\title{
The interaction of two spherical particles in simple-shear flows of yield stress fluids
}

\author{
Mohammadhossein Firouznia $^{\mathrm{a}}$, Bloen Metzger ${ }^{\mathrm{b}}$, Guillaume Ovarlez $^{\mathrm{c}}$, Sarah \\ Hormozi $^{\mathrm{a}, *}$ \\ ${ }^{a}$ Department of Mechanical Engineering, Ohio University, 251 Stocker Center, Athens, \\ Ohio, USA, 45701. \\ ${ }^{b}$ Aix Marseille Universit, CNRS, IUSTI UMR 7343, 13453 Marseille, France. \\ ${ }^{c}$ Univ. Bordeaux, CNRS, Solvay, LOF, UMR 5258, F-33608 Pessac, France.
}

\begin{abstract}
This study focuses on the interaction of two small freely-moving spheres in a linear flow field of yield stress fluids. We perform a series of experiments over a range of shear rates and different shear histories using an original apparatus and with the aid of conventional rheometry, Particle Image Velocimetry (PIV) and Particle Tracking Velocimetry (PTV). We investigate the flow field around a single sphere as well as two spheres in a simple-shear flow. The flow is Stokesian and the Bingham number is in the range of $0 \leq B \leq 2$. To explore the limit of zero Bingham number, we use both Newtonian and shear thinning suspending fluids. We use guar gum solutions and Carbopol gels as shear thinning and yield stress test fluids respectively. We show that the presence of a slight elasticity, which is unavoidable when dealing with polymer solutions, plays an important role in establishing the flow field, e.g., disturbance velocities and stream lines around a single sphere as well as particle trajectories. Therefore, ideal yield stress fluid models cannot provide a full description of flow problems involving particles in practical yield stress fluids. The flow field around a single sphere can be used to understand the two particle interactions. We show how particleparticle contact and non-Newtonian behaviors result in relative trajectories with fore-aft asymmetry. Particularly, the fore-aft asymmetry depends on the Deborah number, Bingham number, shear history, initial offset and roughness of the particles. Finally, we discuss how the relative particle trajectories may affect the microstructure of complex suspensions and consequently the bulk rheology.
\end{abstract}

Keywords: Yield stress materials, PIV visualization, Low-Reynolds-number flows, Simple-shear flow, Elastoviscoplastic materials, Noncolloidal yield stress suspensions

\footnotetext{
${ }^{*}$ Corresponding author

Email address: hormozi@ohio.edu (Sarah Hormozi)
} 


\section{Introduction}

The flows of non-Newtonian slurries, often suspensions of noncolloidal particles in yield stress fluids, are ubiquitous in many natural phenomena (e.g. flows of slurries, debris and lava) and industrial processes (e.g. waste disposal,

5 concrete, drilling muds and cuttings transport, food processing). Studying the rheological and flow behaviors of non-Newtonian slurries is therefore of high interest. The bulk rheology and macroscopic properties of noncolloidal suspensions are related to the underlying microstructure, i.e., the arrangement of the particles. Therefore, investigating the interactions of particles immersed 10 in viscous fluids is key to understanding the microstructure, and consequently, to refine the governing constitutive laws of noncolloidal suspensions. Here, we study experimentally the interaction of two particles in shear flows of yield stress fluids.

There exists an extensive body of research on hydrodynamic interactions of two particles in shear flows of Newtonian fluids. One of the most influential studies on this subject is performed by Batchelor and Green 1 who then used the knowledge of two particle trajectories and stresslets to scale up the results and provide a closure for the bulk shear stress in a dilute noncolloidal suspension 20 to the second order of solid volume fraction, $\phi[2$. Moreover, they showed that due to the fore-aft symmetry of the particle trajectories, Stokesian noncolloidal suspensions do not exhibit any normal stress difference.

The work of Batchelor and Green was followed by subsequent attempts 25 [3, 4, 5, 6] to develop accurate functions describing the hydrodynamic interactions between two particles, which built a foundation for further analytical studies [7, 8, 9, and powerful simulation methods such as Stokesian Dynamics [10. A large body of theoretical and numerical studies has been done to solve the relative motion of two spherical particles in order to obtain the quantities

30 required for the calculation of the bulk parameters, such as mean stress and viscosity in suspensions with a wide range of solid fractions (dilute to semi-dilute) 1, 11, 12, 13, 14 .

The Stokes regime without any irreversible forces leads to symmetric particle 35 trajectories, and consequently, a symmetric Pair Distribution Function (PDF), i.e., the probability of finding a particle at a certain position in space with respect to a reference particle. These result in a Newtonian bulk behavior without any development of normal stress differences in shear flows. However, even in Stokesian suspensions the PDF is not symmetric [15, 16, 8, 17, 18] and the loss

40 of symmetry can be related to contact, due to roughness [19, 20, 21, 22] or other irreversible surface forces (e.g., repulsive force leads to an asymmetric PDF in a similar fashion to how a finite amount of Brownian motion does [8]).

The microstructure affects the macroscopic properties of noncolloidal sus45 pensions leading to non-Newtonian effects (i.e., normal stress differences) and 
phenomena such as shear induced migration of particles [23, 24, 25, 26]. Thus, the development of accurate constitutive equations requires considering the connection between the microstructure and macroscopic properties either explicitly [27, 28, 29] or implicitly through the particle phase stress [30, 31, 32, 23, 33.

A yield stress fluid deforms and flows when it is subjected to a shear stress larger than its yield stress. In ideal yield stress models, such as the Bingham or Herschel-Bulkley models [34, the state of stress is undetermined when the shear stress is below the yield stress and the shear rate vanishes. In the absence 55 of inertia, the solutions to flows of ideal yield stress fluids have the following features: (i) uniqueness (ii) nonlinearity of the equations (iii) symmetries of the domain geometry, coupled methodologically with reversibility and reflection of solutions [35. Therefore, flows around obstacles, such as spheres, should lead to symmetric unyielded regions and to symmetric flow lines in the yielded regions, as observed in simulations [36, 37, 38, 39, 40.

However, recent studies report on phenomena such as loss of fore-aft symmetry under creeping condition and formation of negative wake behind particles, which cannot be explained with the assumption of ideal yield stress fluid [41, 42.

${ }_{65}$ While these behaviors have been attributed to the thixotropy of the material previously [43, recent simulations show similar behaviors for nonthixtropic materials when elastic effects are considered [44, 45]. Therefore, elastoviscoplastic (EVP) models are proposed which consider the contribution of elastic, plastic and viscous effects simultaneously in order to analyze the material behavior 70 more accurately [46, 47, 48].

The field of inclusions (i.e. solid particles, fluid droplets and air bubbles) in yield stress fluids is not as advanced as that of Newtonian fluids. The main challenges are due to the nonlinearity of the constitutive laws of yield stress 75 fluids and resolving the structure of unyielded regions, where the stress is below the yield stress (for more details see [49]). To locate the yield surfaces that separate unyielded from yielded regions, two basic computational methods are used: regularization and the Augmented Lagrangian (AL) approach [37]. On the experimental front, techniques such as PIV [43, 41, 50, 151, 42, [52, PTV ${ }_{80}$ 51, 42, Nuclear Magnetic Resonance (NMR) 53, 54, X-ray [55, 56, Magnetic Resonance Imaging (MRI) [57] are used to study the flow field inside the yielded region as well as determining the yield surface.

Generally speaking, studies of single and multiple inclusions (i.e., rigid par85 ticles and deformable bubbles and droplets) in yield stress fluids are abundant. These studies mainly focus on resolving important physical features when dealing with yield stress suspending fluids, e.g. buoyant inclusions can be held rigidly in suspensions [58, 59, 60, 61, 62, 63, 64, 65, 66; multiple inclusions appear not to influence each other beyond a certain proximity range 61; flows 90 may stop in finite time 66; itc. Other studies exist which address the drag closures, the shape of yielded region, the role of slip at the particle surface and 
its effect on the hydrodynamic interactions [40, 67, 42, 44].

Progressing beyond a single sphere and tackling the dynamics of multiple

particles in a Lagrangian fashion is a much more difficult task. Therefore, another alternative is to address yield stress suspensions from a continuum-level closure perspective. The fundamental objective is then to characterize the rheological properties as a function of the solid volume fraction $(\phi)$ and properties of the suspending yield stress fluid. Recent studies show that adding particles to a yield-stress fluid usually induces an enhancement of both yield stress and effective viscosity while leaving the power-law index intact $[68,69,70,71,72,73,54$.

Unlike the case of settling of particles in yield stress fluids, no attention has been payed to the study of pair interactions of particles in simple flows of yield stress fluids. Our knowledge about this fundamental problem is essential to form a basis for further studies regarding the suspensions of non-Brownian particles in yield stress fluids. To this end, we present an experimental study on the interaction of two small freely-moving spheres in a Couette flow of a yield stress fluid. Our main objective is to understand how the nonlinearity of the suspending 110 fluid affects the particle trajectories, and consequently, the bulk rheology. This paper is organized as follows. Section 2 describes the experimental methods, materials and particles used in this study along with the rheology of our test fluids. In Section 3, we present our results on establishing a linear shear flow in the absence of particles, flow around one particle and the interaction of parti115 cle pairs in different fluids including Newtonian, yield stress and shear thinning. Finally, we discuss our conclusions and suggestions for future works in Section 4

\section{Experimental methods and materials}

In this section we describe the methodology and materials used in this study.

\subsection{Experimental set-up}

The schematic of the experimental set-up is shown in Fig. 1. It is designed to produce a uniform shear flow within the fluid enclosed by a transparent belt. The belt is tightened between two shafts one of which is coupled with a precision rotation stage (M-061.PD from PI Piezo-Nano Positioning) with high angular resolution $\left(3 \times 10^{-5} \mathrm{rad}\right)$ while the other shaft rotates freely. The rotation generated by the precision rotation stage drives the belt around the shafts and hence, applies shear to the fluid maintained in between. In order to have the maximum optical clarity along with the mechanical strength to afford the tension, Mylar sheets (polyethylene terephthalate films from Goodfellow Corporation) of 0.25 $m m$ thickness are used to make the belt. The set-up is designed to reach large enough strains $(\gamma \simeq 45)$ to ensure the steady-state condition. The design is inspired by Rampall et al. 222] and the Couette apparatus is the same as that 


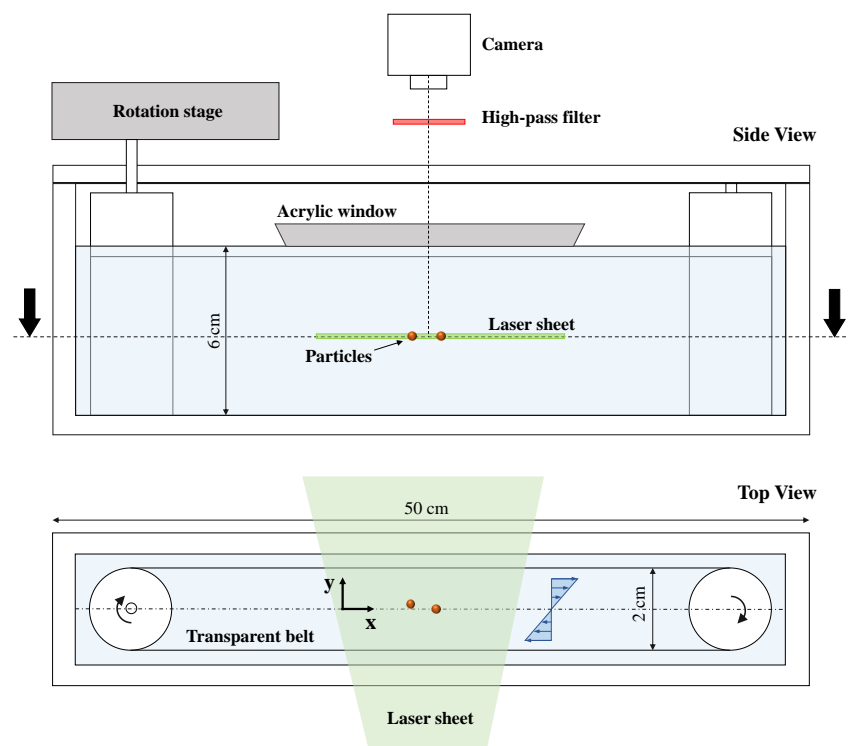

Figure 1: Schematic of the planar Couette-cell and the imaging system: the left shaft is driven by a precision rotation stage while the right shaft rotates freely. Walls are made from transparent acrylic, which allows laser to illuminate the flow field (styled after Fig. 1 of 74])

used by Metzger and Butler in 74.

The flow field is visualized in the plane of shear ( $x y$ plane) located in the mid-plane between the free surface and bottom of the cell. A fraction of the whole flow domain is illuminated by a laser sheet, which is formed by a line generator mounted on a diode laser $(2 \mathrm{~W}, 532 \mu \mathrm{m})$. Fluid is already seeded homogeneously with fluorescently labeled tracer particles, which reflect the in140 cident light (see Sec 2.2). Tracer particles should be small enough to follow the flow field without any disturbance and large enough to reflect enough light needed for image recording. The thickness of the laser sheet is tuned to be around its minimum in the observation window with a plano-convex cylindrical lens. Images are recorded from the top view via a high quality magnification lens (Sigma APO-Macro-180 mm-F3.5-DG) mounted on a high-resolution digital camera (Basler Ace acA2000-165um, CMOS sensor, $2048 \times 1080$ pixel $^{2}, 8$ bit). The reflected light is filtered with a high-pass filter $(590 \mathrm{~nm})$ through which the direct reflection (from the particle surface) is eliminated. A transparent window made of acrylic is carefully placed on the free surface of the fluid in order to eliminate the deformation of the fluid surface and by this, the quality of images is improved significantly. The imaging system is illustrated schematically in Fig. 1. 


\subsection{Particles}

$155 \quad$ Particles used in this study are transparent and made of PMMA (polymethyl methacrylate, Engineering Laboratories Inc.) with radius of $a=1 \mathrm{~mm}$, density of $1.188 \mathrm{gr} / \mathrm{cm}^{3}$ and refractive index of 1.492 at $20^{\circ} \mathrm{C}$. They are dyed with Rhodamine 6G (Sigma-Aldrich) which enables us to preform PTV and PIV at the same time. In order to dye particles the procedure proposed by Metzger and Butler in 74] is followed; PMMA particles are soaked for 30 minutes in a mixture of $50 \%$ wt. water and $50 \%$ ethanol with a small amount of Rhodamine $6 \mathrm{G}$ maintained at $40^{\circ} \mathrm{C}$. They are rinsed with an excess amount of water afterwards to assure there is no extra fluorescent dye on their surface and the coat is stable. The surface of the particles from the same batch have been previously observed by Phong [75, 21] and Souzy [76] using Atomic Force Microscope (AFM) and Scanning Electron Microscope (SEM). The root mean square and peak values of the roughness are measured to be $0.064 \pm 0.03 \mu \mathrm{m}$ and $0.6 \pm 0.3 \mu \mathrm{m}$ respectively after investigating an area of $400 \mu \mathrm{m}^{2}$ [21]. Moreover, in order to perform PIV, the fluid is seeded with melamine resin particles dyed with Rhodamine B with

170 a diameter of $3.87 \mu m$, provided by Microparticle $\mathrm{GmbH}$.

\subsection{Fluids}

In this study, three different fluids have been used including Newtonian, yield stress and shear thinning fluid; each of the fluids is described in the following 175 sections:

\subsubsection{Newtonian fluid}

The Newtonian fluid is designed to have the density and refractive index (RI) matched with that of the PMMA particles. Any RI mismatch could lead to refraction of the laser light when it passes the particle-fluid interface which decreases the quality of the images and makes the post processing very difficult or even impossible. However, we only have one or two particles in our experiments and therefore, a slight refractive index mismatch does not result in a poor quality image. The fluid consists of $76.20 \%$ wt. Triton X-100, $14.35 \%$ wt. 185 of zinc chloride, $9.31 \%$ wt. of water and $0.14 \%$ wt. of hydrochloric acid [77] with the viscosity of $4.64 \mathrm{~Pa} . \mathrm{sec}$ and refractive index of $1.491 \pm 10^{-3}$ at room temperature. A small amount of hydrochloride acid prevents the formation of zinc hyperchlorite and thus enhances the transparency of the solution. Water is first added to zinc chloride gradually and the solution is stirred until all solid 190 particles dissolve in the water. Since the process is exothermal we let the solution cool down to reach room temperature. After adding hydrochloride acid to the cooled solution, Triton X-100 is added and mixed until the final solution is homogeneous. 


\subsubsection{Yield stress fluid} tical static and dynamic yield-stress independent of the flow history 78, 79. To this end, we chose Carbopol 980 which is a cross-linked polyacrylic acid with high molecular weight and is widely used in the industry as a thickening agent. Most of the experimental works studying the flow characteristics of the thixotropy can be neglected. Carbopol 980 is available in a form of anhydrous solid powder with micrometer sized grains. When mixed with water, polymer chains hydrate, uncoil and swell forming an acidic solution with $\mathrm{pH} \sim 3-4$. When neutralized with a suitable basic agent such as sodium hydroxide, mijam (depending on the concentration) forming a structure which exhibits yieldstress and elastic behavior 80, 81. Rheological properties of Carbopol gels are dependent of both concentration and $\mathrm{pH}$. At intermediate concentrations, both yield-stress and elastic modulus increase with $\mathrm{pH}$ until they reach their peak hensive study on the microstruture and properties of Carbopol gel is provided by Piau in 82 .

In order to make Carbopol gel with a density matched with that of PMMA and glycerol $72.17 \%$ wt (provided by ChemWorld) is prepared, which has the same density as the PMMA particles. Then, depending on the concentration needed for the experiment (varies in the range of 0.07-0.2 \% wt. in this study), the corresponding amount of Carbopol 980 (provided by Lubrizol Corporation) let to be mixed for hours until all Carbopol particles hydrate and the dispersion is homogeneous. A small amount of sodium hydroxide (provided by SigmaAldrich) is then added in order to neutralize the dispersion. It is suggested to add all of the neutralizer at once, or at least in a short amount of time since 225 as $\mathrm{pH}$ increases the viscosity increases drastically which would increase mixing time. The solution becomes more transparent as it reaches neutral $\mathrm{pH}$. The refractive index of the Carbopol gels used in this study varies in the range of $1.370 \pm 5 \times 10^{-3}$. By investigating the rheological properties of the gel at different $\mathrm{pHs}$, we found $\mathrm{pH}=7.4$ to be a stable point with highest yield-stress and elastic The final solution is transparent, homogeneous with no visible aggregates. Also, the rheometry results of all samples taken from different parts of the solution batch collapse. The compositions of all Carbopol gels used in this study are described in Table 1

235

\subsubsection{Shear thinning fluid}

In order to investigate the effect of yield-stress and shear thinning individually, it is required to study the problem with a shear thinning fluid with no 
yield stress. Therefore, we chose Hydroproxypyl Guar which is a derivative of

(provided by Solvay Inc.) is used in this study which is widely used in cosmetics and personal care products 83. It is transparent when mixed with water and exhibits negligible yield stress in low to moderate concentrations. The refractive index of the guar gum solutions used in this study varies in the range of $245 \quad 1.368 \pm 5 \times 10^{-3}$.

In order to make a solution of Jaguar HP-105 with the same density as the particles, we follow the same scheme mentioned earlier for Carbopol gel in sec 2.3.2. First, a solution of deionized water $27.83 \%$ wt. and glycerol $72.17 \%$ wt (provided by ChemWorld) is prepared. While being mixed by a mixer, depending on the desirable concentration (in this study varies from $0.3-0.6 \%$ wt.), corresponding amount of Jaguar HP-105 is added gradually to the solution. The dispersion is covered and mixed for 24 hours until a homogeneous solution is achieved. Homogeneity is tested by comparing rheometry results performed on guar gum solutions used in this study are described in Table 1.

\subsection{Rheometry}

Unlike Newtonian fluids, the effective viscosity of the non-Newtonian fluids depends on the shear rate and flow history. Here, we explain the rheological tests performed to characterize the non-Newtonian behaviors. For each test the procedure is described followed by the results and the interpretation. All measurements shown in this section are carried out using serrated parallel plates with a stress-controlled DHR-3 rheometer (provided by TA Instruments) on samples of Carbopol gels and guar gum solutions referred to as "YS1-2" and "ST" respectively. The rheological properties of all test fluids used in this study are described in Table.1.

A logarithmic shear rate ramp with $\dot{\gamma} \in[0.001,10] \mathrm{sec}^{-1}$ is applied on samples of test fluids for a duration of $105 \mathrm{sec}$ in order to find the relation between shear rate and shear stress, $\tau=f(\dot{\gamma})$ (see Fig. 2). During the increasing shear ramp, the material is sheared from rest. The behavior of the yield stress material is hence similar to a Hookean solid until the stress reaches the yield stress. Beyond the yield stress, the material starts to flow like a shear thinning liquid.

275 On the contrary, during the decreasing shear ramp, the yield stress material is already in flow condition and the stress asymptotes to the yield stress at low shear rates (see Fig. 2a). The value of yield stress during both increasing and decreasing ramps are identical. This is the typical behavior of non-thixotropic yield-stress materials (more information can be found in [84, 85]). The measurements of increasing and decreasing ramps overlap beyond yield stress and show no sign of hysteresis. The rheological behavior of Carbopol gel is described well by the Herschel-Bulkley (see Eq. 1) model as shown in Fig. 2. 
Table 1: Composition, $\mathrm{pH}$ and rheological properties of the test fluids used in this study: NWT (Newtonian fluid), YS1-2 (yield stress fluids) \& ST (shear thinning fluid). Dynamic moduli, $G^{\prime}$ and $G^{\prime \prime}$ are measured at $\omega=1 \mathrm{rad} . s e c^{-1}, \gamma_{0}=0.25 \%$.

\begin{tabular}{l|cccc} 
& \multicolumn{4}{|c}{ Test fluids } \\
\hline Materials (\% wt.) & ST & YS1 & YS2 & NWT \\
\hline Water & 71.764 & 71.969 & 75.004 & 9.31 \\
Glycerol & 27.707 & 27.876 & 24.766 & - \\
Carbopol 980 & - & 0.116 & 0.170 & - \\
Jaguar HP-105 & 0.529 & - & - & - \\
Sodium hydroxide & - & 0.039 & 0.060 & - \\
Triton X-100 & - & - & - & 76.20 \\
Zinc chloride & - & - & - & 14.35 \\
Hydrochloric acid & - & - & - & 0.14 \\
\hline pH & - & 7.40 & 7.44 & - \\
\hline$\tau_{y}(P a)$ & 0 & 3.3 & 46.6 & 0 \\
$K(P a . s e c$ & $n$ \\
$n$ & 6.7 & 4.6 & 18.7 & 4.6 \\
\hline$G^{\prime}(P a)$ & 0.46 & 0.50 & 0.30 & 1 \\
$G^{\prime \prime}(P a)$ & 3.5 & 17.9 & 213.5 & - \\
\hline
\end{tabular}




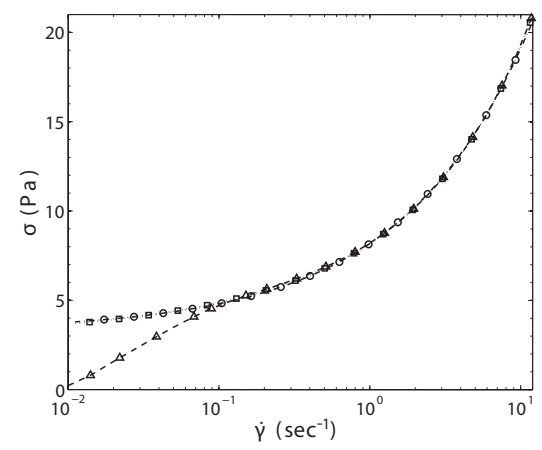

(a)

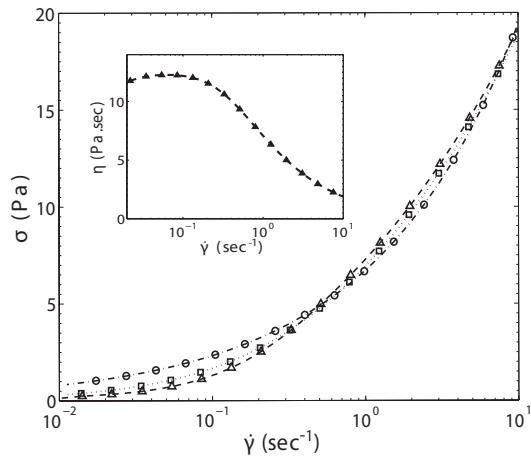

(b)

Figure 2: Stress versus shear rate for a cycle of logarithmic shear rate ramps applied on samples of YS1 (a) and ST (b): increasing ramps $(\Delta)$, decreasing ramps ( $\square$ ) and the corresponding Herschel-Bulkley fits $(\mathrm{O})$ described in the Table 1 . The inset of (b) presents the variation of viscosity versus shear rate for ST.

$$
\tau=\tau_{y}+K(\dot{\gamma})^{n}
$$

285 These values are calculated for YS1-2 in range of $\dot{\gamma} \in[0.01,10] \mathrm{sec}^{-1}$ in Table. 1].

Fig. $2 \mathrm{~b}$ shows the rheology of the guar gum solution, ST in the plane of shear stress versus shear rate. The Carreau-Yasuda model has generally been adopted to explain the rheological behavior of guar gum solutions [86, 87. The inset of Fig. $2 \mathrm{~b}$ shows the viscosity of the guar gum solution versus shear rate following the Carreau-Yasuda model. We see that the viscosity presents a plateau, $\eta_{0} \approx 12.2$ Pa.sec, in the limit of small shear rates, $\dot{\gamma}<0.1 \mathrm{sec}^{-1}$. At

$295 \dot{\gamma}>0.1 \mathrm{sec}^{-1}$ viscosity decreases with shear rate until it reaches another plateau at higher shear rates. Here, we adopt a power-law model which properly describes the rheological behavior of the material in the range of shear rate in our experiments. The values of consistency and power-law index are reported in Table 1 .

Practical yield-stress fluids exhibit viscoelastic behavior as well. Therefore, it is expected that the shear history has an impact on the behavior of the material. We have adopted two experimental procedures to evaluate the effect of shear history. In the first procedure, we shear the material ensuring that the 305 strain is sufficient to break the micro-structure of gel and reach a steady state. Then, we rest the material for one minute (zero stress) and apply the shear in the same direction as the pre-shear (hereafter called positive pre-shear). In 


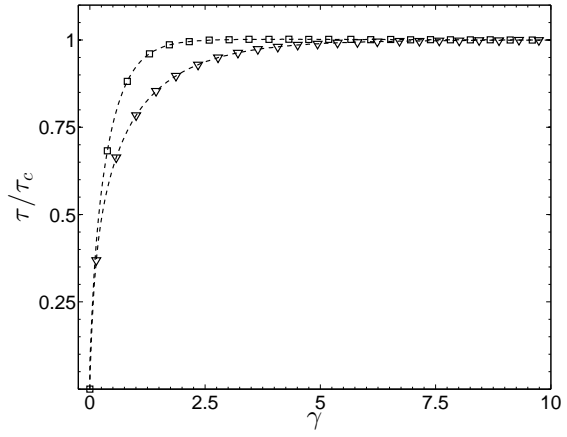

(a)

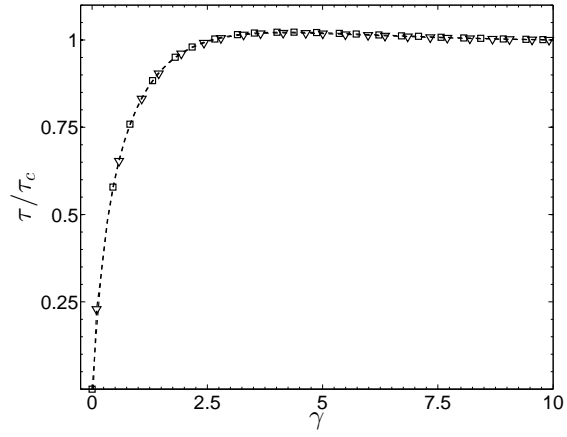

(b)

Figure 3: Normalized stress versus strain for samples of yield stress and shear thinning test fluids under a constant shear rate with different shear histories: (a) YS1 at $\dot{\gamma}=0.129 \mathrm{sec}^{-1}$ $(B, D e)=(2.0,0.09)$, (b) ST at $\dot{\gamma}=0.26 \mathrm{sec}^{-1} D e=1.03$. Triangle markers represent negative pre-shear while square markers indicate positive preshear.

the second procedure, we reverse the direction of the applied shear after imposing a pre-shear on the material (hereafter called negative pre-shear) and a 310 rest period. Fig. 3a shows that under a constant applied shear stress the yield stress material reaches its steady state after a larger strain when negative preshear is applied. However, the shear history does not affect the behavior of the guar gum solution as shown in Fig. 3b. These procedures helped us design the experimental protocol for our Couette flow experiments (see Sec. 3.3.2).

315 One can conclude that a preshear in the same direction as the shear imposed subsequently in the experiments is appropriate for having a behavior close to that of ideal visco-plastic behavior.

In order to characterize the viscoelasticity of the test fluids further, the shear storage modulus, $G^{\prime}$ and the shear loss modulus, $G^{\prime \prime}$ (representing the elastic and viscous behavior of the material respectively) are measured during oscillatory tests. Dynamic moduli of YS1 and ST are shown in Fig. 4 as a function of strain amplitude, $\gamma_{0} \in\left[10^{-1}, 10^{3}\right] \%$ while frequency is constant, $\omega=1{\mathrm{rad} . \mathrm{sec}^{-1}}^{-}$. We observe that the behavior is linear up to $\gamma_{0} \approx 1 \%$ in YS1 while it remains linear 325 at larger strain amplitudes, $\gamma_{0} \approx 10 \%$ in ST. Elastic effects are dominant (i.e. $G^{\prime}>G^{\prime \prime}$ ) at strain amplitudes lower than $\gamma_{0} \approx 100 \%$ in the yield stress material, YS1 (see Fig. 4a). At $\gamma_{0}>100 \%$, the shear loss modulus becomes larger than the shear storage modulus in YS1 indicating that the viscous effects take over. On the other hand, elastic and viscous effects are equally important in ST in the linear viscoelastic regime as the shear loss and shear storage moduli have identical values under $\gamma_{0} \approx 100 \%$ (see Fig. $4 \mathrm{~b}$ ). At larger strain amplitudes however, the shear loss modulus becomes larger implying larger viscous effects. The values of $G^{\prime}$ and $G^{\prime \prime}$ reported in Table 1 are measured at $\omega=1 \mathrm{rad}_{\mathrm{sec}}{ }^{-1}$, $\gamma_{0}=0.25 \%$. In Fig. 5 the variation of dynamic moduli is given as a function of 335 frequency for the Carbopol gel, YS1 and the guar gum solution, ST. Different 


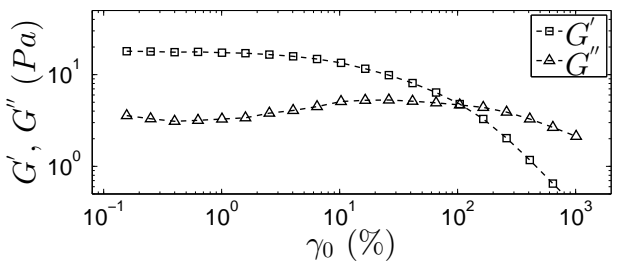

(a)

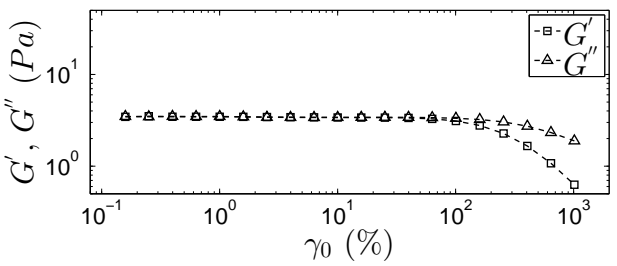

(b)

Figure 4: Elastic and viscous moduli with respect to strain amplitude in strain amplitude sweep tests with an angular frequency of 1 rad.sec ${ }^{-1}$ on samples of YS1 (a), and ST (b). $G^{1}$ starts to decrease beyond a critical strain below which it is nearly constant.

curves correspond to different strain amplitudes $\left(\gamma_{0}=1,5,20,50,100 \%\right)$.

\subsection{Post-processing}

The PMMA particles are tracked during their motion via Particle Tracking 340 Velocimetry (PTV) to extract the trajectories. Images are recorded at strain increments of $\gamma_{r e c} \leq 0.6 \%$ to ensure high temporal resolution. In each image, the center and radius of each particle is detected via the Circular Hough Transform 88,89 . Due to the small strain difference between two respective images and consequently small displacement of PMMA particles, same particles are identified and labeled in two images. Applying this methodology to all images we obtain trajectories of particles.

Particle Image Velocimetry (PIV) is employed to measure the local velocity field from successive images recorded from the flow field. It is worth mentioning 350 that in this method we calculate the two dimensional projection of the velocity field in the plane of shear ( $x y$ plane).

We have used the MatPIV routine with minor modifications in order to analyze PIV image pairs [90. Each image is divided into multiple overlapping sub-images, also known as interrogation windows. The PIV algorithm goes through three iterations of FFT-based cross-correlation between corresponding interrogation widows in two successive images in order to calculate the local velocity field. The velocity field measured in each iteration is used to improve the accuracy during the next iteration where the interrogation size is reduced 


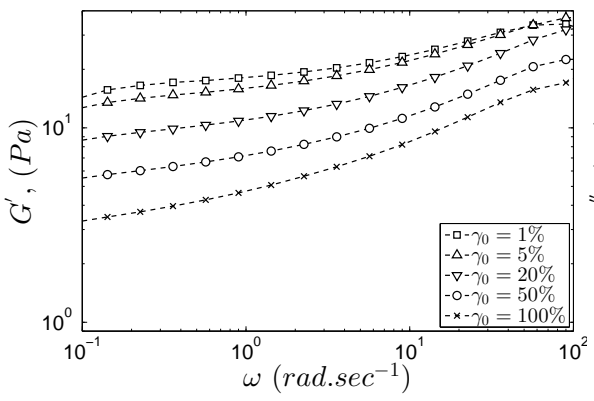

(a)

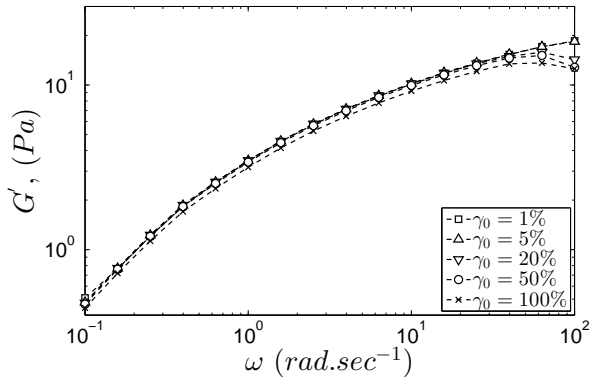

(c)

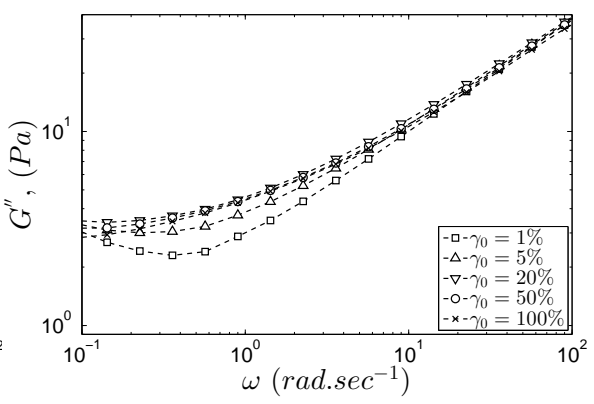

(b)

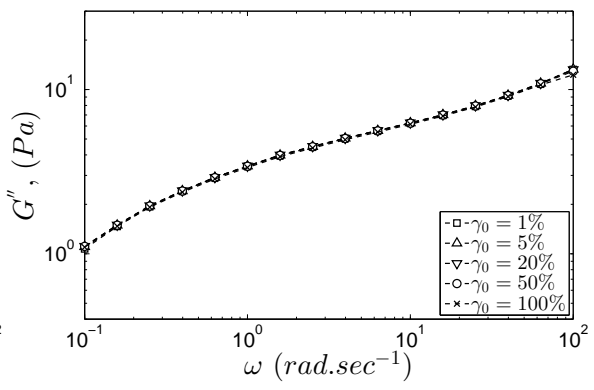

(d)

Figure 5: Dynamic moduli, $G^{\prime}$ (left column) and $G^{\prime \prime}$ (right column) for samples of YS1 (first row) and ST (second row) during frequency sweep from 0.1 to $100 \mathrm{rad} / \mathrm{sec}$. Different markers correspond to different strain amplitudes, $\gamma_{0}=1,5,20,50,100 \%$. 

the overlap of $50 \%$ are selected respectively during the first, second and third iterations. Following each iteration, spurious vectors are identified by different filters such as signal-to-noise ratio, global histogram and local median filters. Spurious vectors are then replaced via linear interpolation between surrounding cant error due to the interpolation process. The measured velocity is ignored if the interrogation window overlaps with the particle surface (detected earlier via PTV algorithm). The size independence of the velocity measurements is verified by comparing the results with that obtained when we increase the interrogation widow size to $32 \times 32$ pixels $(\sim a / 4.5)$.

\section{Experimental results}

\subsection{Establishing a linear shear flow in the absence of particles}

The first step is to establish a linear shear flow field within the experimental Couette-cell affects the flow field around one particle, or the interaction of two particles. Our Couette-cell has a finite dimension bounded with a wall from the bottom, an acrylic window from the top and two rotating cylinders from the sides (see Fig 1). It is essential to show that a linear shear flow is achievable in is defined as:

$$
R e=\frac{4 \rho(2 U / H) a^{2}}{\mu}
$$

which is of the order $O\left(10^{-5}\right)$ in our experiments, implying that the inertial effects are negligible. Here $a$ and $H$ are the particle radius and gap width respectively, $U$ is the maximum velocity across the gap, $\rho$ is the density and Couette-cell (50 cm long versus $2 \mathrm{~cm}$ wide), the central region where measurements are made is far from the shafts. In the absence of inertia and boundary effects the solution to the momentum equations would give us a linear velocity profile in our configuration, independent of the rheology of the test fluids. In this section, we present our experimental results showing how a linear shear flow field is established within the Couette-cell when we have different suspending fluids including Newtonian fluids, yield stress fluids and shear thinning fluids.

In the case of the Newtonian fluid, Fig 6a shows the velocity profile across 395 the gap for different shear rates imposed at the belt. The velocity field is averaged along the $x$-direction (flow direction). We normalize the velocity with the maximum velocity across the PIV window, $u_{c}$, and show that all velocity profiles collapse to a master curve (see Fig. 6b.). A linear shear flow is achieved 


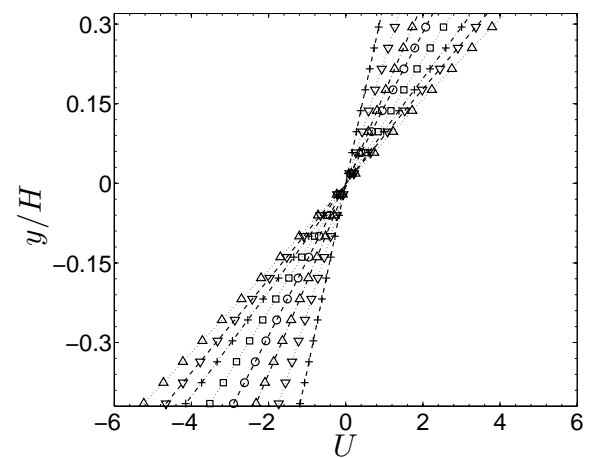

(a)

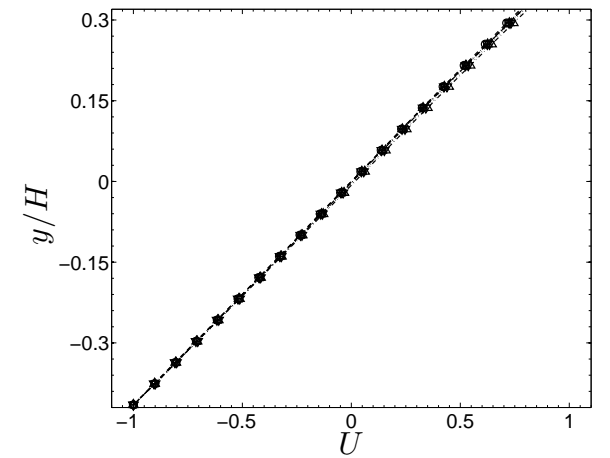

(b)

Figure 6: (a) Velocity profiles averaged along the x-direction for the Newtonian fluid when subjected to different shear rates of $\dot{\gamma}=0.18,0.26,0.35,0.44,0.52,0.61,0.70,0.79 \mathrm{sec}^{-1}$. (b) Normalized velocity profiles across the gap.

consequently with the Newtonian fluid.

400

When we deal with a yield stress test fluid, there exist more dimensionless numbers in addition to the bulk Reynolds number including Bingham number $(B)$ which is the ratio of yield stress $\left(\tau_{Y}\right)$ to the viscous stress $\left(K \dot{\gamma}^{n}\right)$ in the flow:

$$
B=\frac{\tau_{Y}}{K \dot{\gamma}^{n}}
$$

405 Another important dimensionless number is Deborah number which is the ratio of the material time scale to the flow time scale. For elastoviscoplastic materials, the relaxation time $\lambda$, the elastic modulus $G^{\prime}$, and the apparent plastic viscosity $\eta_{p}$ are related via $\eta_{p}=\lambda G^{\prime}$ where the so-called plastic viscosity is defined as follows [44]:

$$
\eta_{p}=\frac{\tau-\tau_{Y}}{\dot{\gamma}}
$$

${ }_{410}$ Comparing Eq. (4) with (1), we conclude $\eta_{p}=K \dot{\gamma}^{n-1}$. Therefore, the Deborah number is:

$$
D e=\lambda \dot{\gamma}=\frac{K \dot{\gamma}^{n}}{G^{\prime}}
$$

Velocity fields obtained via PIV measurements are averaged along the flow direction. Fig. 7 a shows the measured velocity profiles across the gap when normalized by the maximum velocity across the PIV window, $u_{c}$. Next, shear rate profiles are calculated from the averaged velocity profiles according to Eq. (6) and are used to calculate the shear stress profiles via the Herschel-Bulkley model (shown in Fig. $7 \mathrm{~b}$ and $7 \mathrm{c}$ respectively). Shear rate profiles are normalized by the average shear rate across the gap $\dot{\gamma}_{c}$, while stress profiles are normalized 


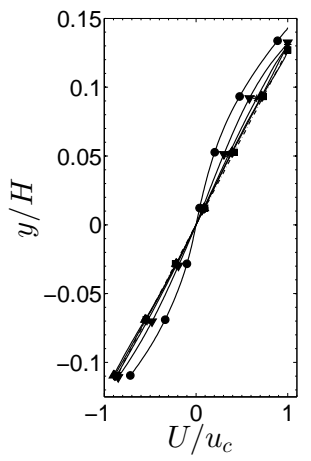

(a)

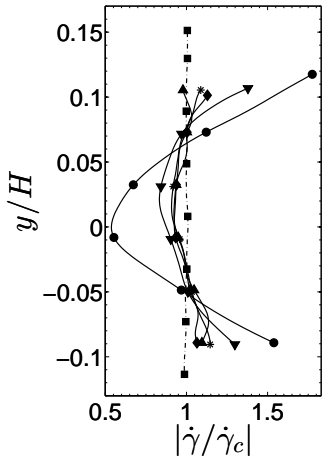

(b)

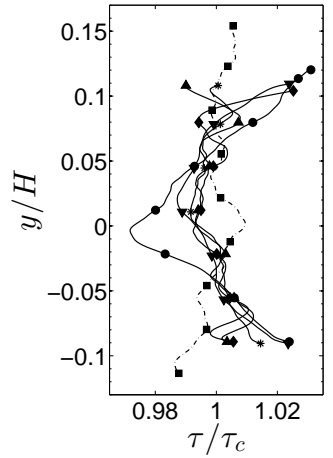

(c)

Figure 7: (a) Normalized velocity profiles across the gap when YS2 undergoes shear flows at different Bingham numbers: $(B, D e)=(4.6,0.05)(\bullet),(B, D e)=(3.2,0.07)(\boldsymbol{\nabla}),(B, D e)=$ $(2.3,0.10)(*),(B, D e)=(2.2,0.10)(\mathbf{\Delta}),(B, D e)=(2.0,0.11)(\bullet)$ compared to that of the Newtonian fluid, NWT (ם). (b) The corresponding dimensionless shear rate profiles and (c) stress profiles.

by the average stress across the gap $\tau_{c}$.

$$
\dot{\gamma}_{l o c}=\sqrt{2\left(\frac{\partial u_{d}}{\partial x}\right)^{2}+2\left(\frac{\partial v_{d}}{\partial y}\right)^{2}+\left(\frac{\partial u_{d}}{\partial y}+\frac{\partial v_{d}}{\partial x}\right)^{2}}
$$

It is evident that as we increase the Bingham number, the velocity profile deviates from a linear shape, and consequently, the shear rate is not constant. This is quite a unique observation for a yield stress fluid, and the rheology of the fluid can explain this puzzle. Let us take a closer look at the variation of stress with respect to the shear rate shown in Fig. 2 for the yield stress test fluids used in the experiments. We can see that at low shear rates (i.e. high Bingham numbers), such as $0.01<\dot{\gamma}<0.1 \mathrm{sec}^{-1}$, a small variation in the shear stress projects to a large variation in the shear rate. On the contrary, at higher shear rates $\dot{\gamma}>1 \mathrm{sec}^{-1}$ (i.e. low Bingham numbers) the same amount of stress variation corresponds to a significantly smaller variation in the shear rate. Fig. $7 \mathrm{c}$ shows the variation of stress across the gap is of the same order for all Bingham numbers while the resulting shear rate profiles are significantly different in terms of inhomogeneity. This implies that a small stress inhomogeneity due to any imperfection of the set-up and the test fluid (finite dimension of the set-up, slight inhomogeneity in the test fluid or etc.) projects into a larger shear rate inhomogeneity as we increase the Bingham number. This stress inhomogeneity is estimated from Fig. $7 \mathrm{c}$ to be $\approx 2 \%$ in our set-up.

Both the characteristic length of the inhomogeneity and its amplitude increase as the Bingham number increases. Our results show that for $B \leq 2$, the shear rate inhomogeneity is minimal (comparable to that of the Newtonian test fluid), and we can establish a linear velocity profile in the set-up for the case of 
a yield stress fluid. Therefore, all the experiments in this work are performed

\subsection{One particle in a linear shear flow}

This section is aimed at studying a linear shear flow around one particle in the limit of zero $R e$ when we have different types of fluids including Newtonian, 450 yield stress and shear thinning. A theoretical solution is available for a particle in a Newtonian fluid subjected to a linear shear flow field. We use the theoretical solution to validate our experimental results. The effect of a non-Newtonian fluid on the flow field around one particle is then investigated experimentally. Studying the disturbance fields around one particle is key to understanding the hydrodynamic interaction of two particles, and consequently, the bulk behavior of suspensions of noncolloidal particles in non-Newtonian fluids.

\subsubsection{Stokes flow around one particle in a linear shear flow of a Newtonian} fluid: comparison of theory and experiment

First, we compare our PIV measurements with the available theoretical solution for the Stokes flow around one particle in a linear shear flow of a Newtonian fluid 91. The normalized velocity field obtained via a theoretical solution is illustrated in Fig. 8a along with the measured velocity field via PIV in Fig. 8b. which is normalized by the velocity at the belt. A quantitative comparison is given in Figs. $8 \mathrm{~d}, 8 \mathrm{f}$ where dimensionless velocity profiles are compared at cross It is noteworthy to mention that the PIV measurements are available at distances $r / a \geq 1+\epsilon$, where $r$ is the distance from the particle center and $\epsilon \sim 0.1$ is given by the resolution of the PIV interrogation window. The close agreement between our velocity measurements with that predicted by the theory allows 470 us to employ our method for the case yield stress fluids, where the theoretical solution is unavailable. Our experimental data can be used as a benchmark for these fluids.

\subsubsection{Creeping flow around one particle in a linear shear flow: Newtonian and non-Newtonian suspending fluids}

We present our PIV measurements of creeping flows around one particle in linear shear flows of Newtonian, shear thinning (guar gum solution) and yield stress (Carbopol gel) suspending fluids. About 100 PIV measurements (i.e., 100 PIV image pairs) are averaged afterwards to reduce the noise. The origin of the coordinate system, $(x, y, z)$, is fixed on the center of the particle and 480 translates with it (non-rotating). We subtract the far field velocity profile from the experimentally-measured velocity field in order to calculate the disturbance velocity field around one particle:

$$
\mathbf{u}_{d}=\left(u_{d}, v_{d}\right)=\mathbf{u}-\mathbf{u}_{\infty}
$$

Where $u_{d}$ and $v_{d}$ are components of the disturbance velocity vector along the flow direction and gradient direction respectively. The disturbance velocity field 


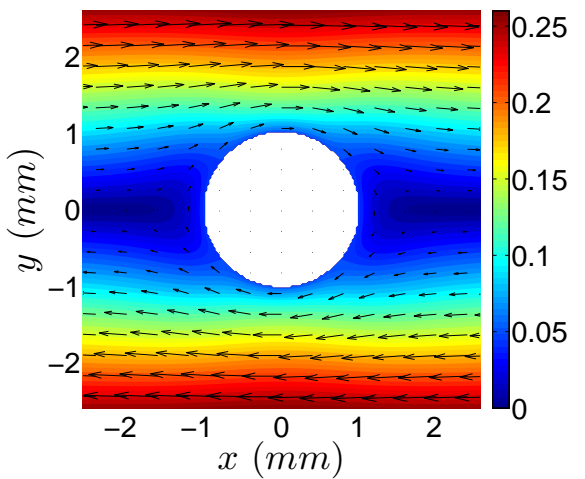

(a)

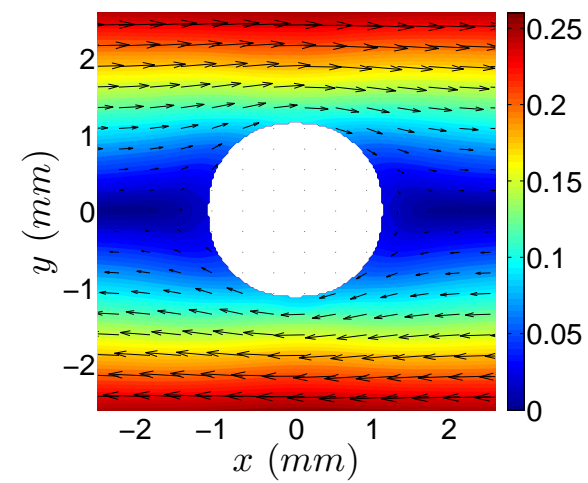

(b)

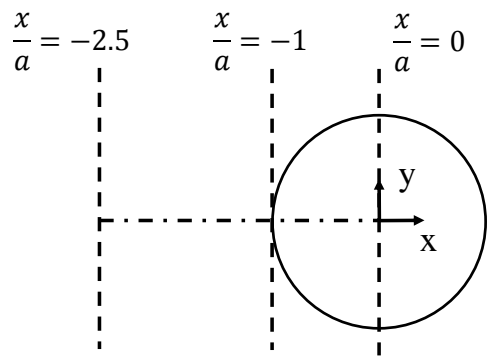

(c)

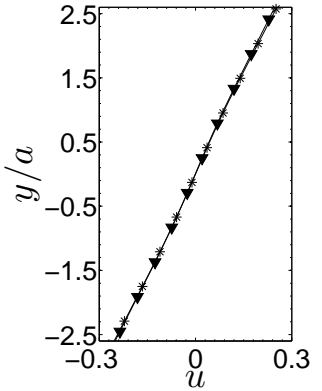

(d)

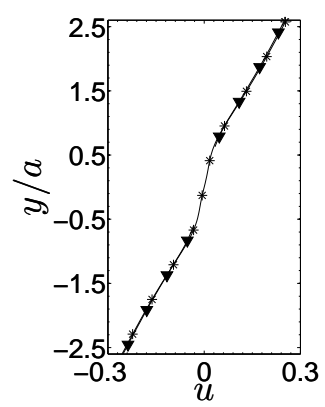

(e)

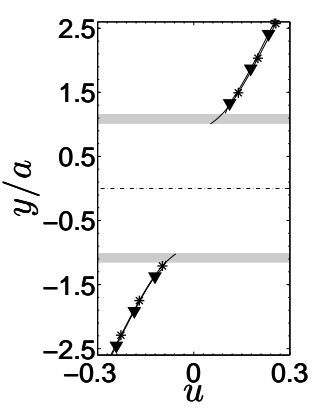

(f)

Figure 8: (a) Normalized velocity field obtained via theoretical solution for a Newtonian fluid. (b) Normalized velocity field for the Newtonian fluid NWT measured via PIV at $\dot{\gamma}=0.27$ $\sec ^{-1}$. (c) Schematic of the particle and locations where velocity profiles are compared with the theory. (d-e) Comparison between velocity profiles obtained from theory $(*)$ and experimental measurements $(\boldsymbol{\nabla})$ at different locations. 


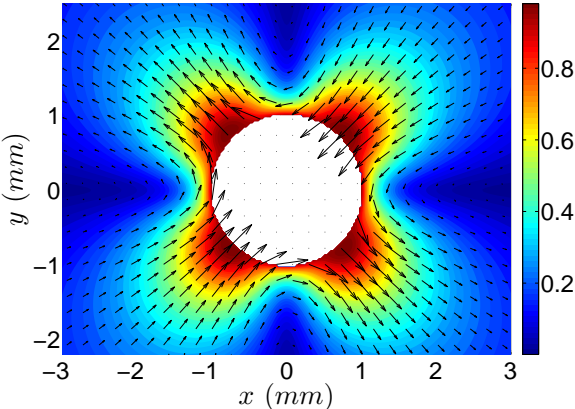

(a)

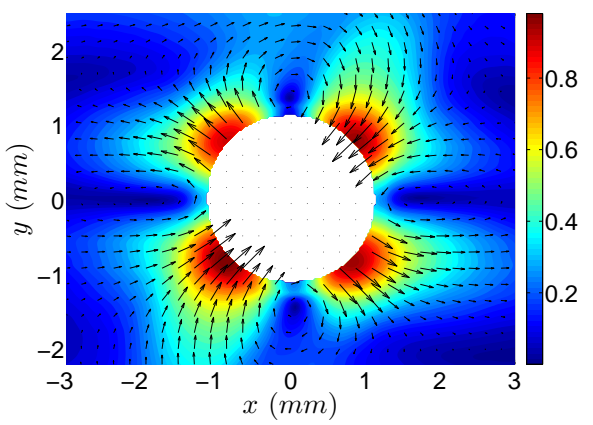

(c)

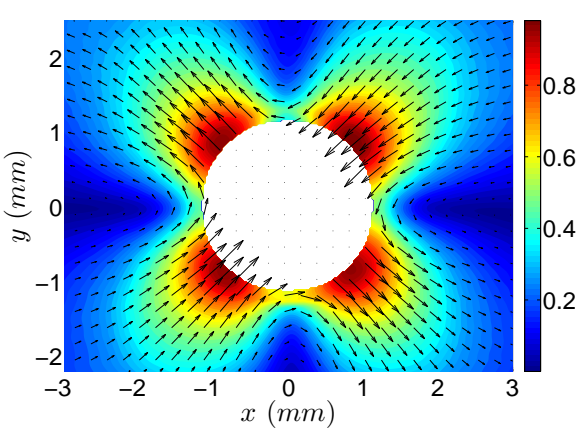

(b)

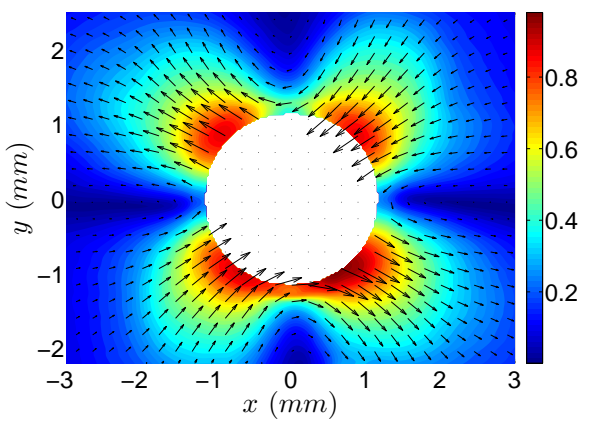

(d)

Figure 9: Normalized disturbance velocity fields around one particle in the shear flow of different fluids: (a) theoretical solution for a Newtonian fluid, (b) experimental results for a Newtonian fluid at $\dot{\gamma}=0.27 \mathrm{sec}^{-1}$, (c) experimental results for the Carbopol gel, YS1 at $\dot{\gamma}=0.34 \mathrm{sec}^{-1}(B, D e)=(1.23,0.15)$, (d) experimental results for the guar gum solution, $\mathrm{ST}$ at $\dot{\gamma}=0.26 \mathrm{sec}^{-1} \mathrm{De}=1.03$.

485 is then normalized by the maximum disturbance velocity in the PIV window.

Fig. 9 shows the normalized disturbance velocity field around one particle in linear shear flows of a Newtonian fluid (theory: Fig. 9a and experiment: Fig. 9b), a yield stress fluid (experiment of Carbopol gel: Fig. 9c) and a shear 490 thinning fluid (experiment of guar gum solution: Fig. 9d). The shear flow is established as $\mathbf{u}=(\dot{\gamma} y, 0,0)$ where $\dot{\gamma}>0$. The disturbance velocity field is normalized by the maximum disturbance velocity in the field. Although the theoretical solution for the case of a single rigid sphere in a simple-shear flow of a Newtonian fluid exists, there is no theoretical solution in the case of a yield 495 stress fluid. Therefore, our experimental measurements shown in Fig. 9c serves as the first set of information about simple-shear flows around a spherical particle.

Fig. 10 shows the colormaps of shear rate around one particle in linear shear 500 flows of a Newtonian fluid (theory: Fig. 10a and experiment: Fig. 10b), a yield stress fluid (experiment of Carbopol gel: Fig. 10c) and a shear thinning fluid 


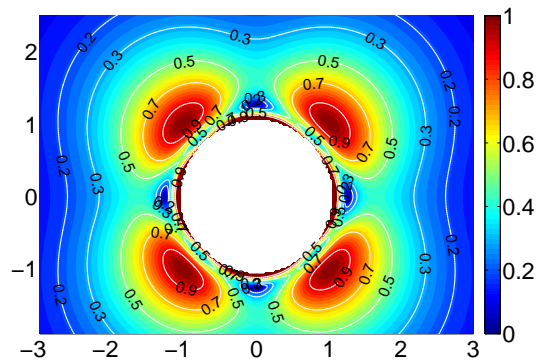

(a)

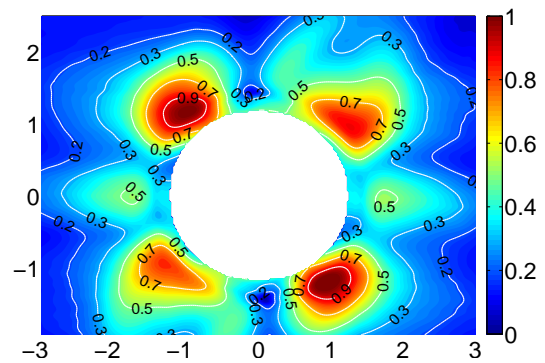

(c)

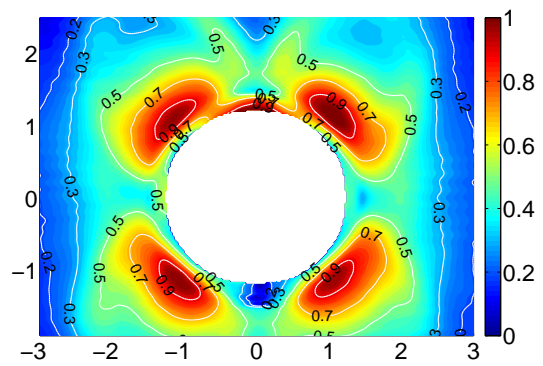

(b)

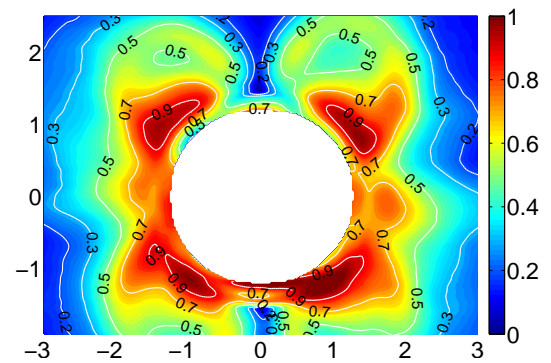

(d)

Figure 10: Normalized shear rate fields around one particle in the shear flow of different fluids: (a) theoretical solution for a Newtonian fluid, (b) experimental results for a Newtonian fluid at $\dot{\gamma}=0.27 \mathrm{sec}^{-1}$, (c) experimental results for the Carbopol gel, YS1 at $\dot{\gamma}=0.34 \mathrm{sec}^{-1}$ $(B, D e)=(1.23,0.15),(d)$ experimental results for the guar gum solution, ST at $\dot{\gamma}=0.26$ $\sec ^{-1} \mathrm{De}=1.03$.

(experiment of guar gum solution: Fig. 10d). The magnitude of local shear rates are calculated by taking the spatial derivative of the disturbance velocity fields based on Eq. (6). Although taking the derivative of experimental data (i.e., PIV measurements of the velocity field) amplifies the noise, averaging over more than 100 PIV measurements reduces the noise and allows us to see the qualitative features.

For the Newtonian fluid, our experimental results shown in Fig. 9b are in a very close agreement with the theoretical solution illustrated in Fig. 9a, we can see that the disturbance velocity has fore-aft symmetry and decays as we move away from the particle surface. Unlike the Newtonian fluid, fore-aft symmetry is broken for our non-Newtonian test fluids (see Fig. 9c and 9d). The fore-aft asymmetry is significantly larger for the Carbopol gel (in Fig. 9c). As men515 tioned in Section 11, the loss of fore-aft symmetry is not predicted for the flow field around one particle if we use ideal visco-plastic constitutive models; e.g. Herschel-Bulkley and Bingham models [36, 37, 38, 39. However, practically speaking, both the guar gum solution and Carbopol gel are polymer based solutions with slight elasticity, and consequently, these are not ideal visco-plastic fluids. Elastic effects are thus responsible for the fore-aft asymmetry observed 
in Fig. 9c and 9d. For viscoelastic fluid flows, uniqueness and nonlinearity are present but the symmetry and reversibility are missing. We should mention that by adopting an appropriate pre-shear procedure in our experiments (described in Section 2.4), we eliminated the possible effects due to the shear history.

Despite the loss of fore-aft symmetry which is evident in Fig. 9c and Fig. 9d, we note that the velocity disturbance field is symmetric with respect to the center of the particle (symmetric with respect to a point). This is indeed expected. Assume two fluid elements are moving towards the particle and located at the ${ }_{530}$ top left and bottom right of the flow field, but at the same vertical distance from the particle. Both fluid elements experience the same shear history during their motion (e.g., compression, extension, rotation) resulting in a symmetric flow field with respect to the center of the particle. The Deborah number is calculated based on the values of shear storage moduli measured at the frequency $535 \omega=1{\mathrm{rad} . \mathrm{sec}^{-1}}^{-1}$ with low strain amplitudes, $\gamma_{0}=0.25 \%$ (see Table 1). In the experiment with the Carbopol gel, YS1 (Fig. 9c) the Deborah number is $D e=0.15$ while it is $D e=1.03$ in the case of guar gum solution, ST(Fig. 9d). Although the Deborah number is relatively small in our experiments, it clearly affects the flow field around the particles. This is consistent with the results 540 of Fraggedakis et al. 44, where they observed the effect of slight elasticity in a yield stress fluid to be significant in establishing the flow field around a single particle settling in a stationary column of a yield stress fluid. Despite the smaller value of the $D e$ number for the case of Carbopol gel compared to the guar gum solution, we see that the fore-aft asymmetry is larger. It can be due the interplay between plastic and elastic effects in the Carbopol gel which is an elasoviscoplastic material. Further investigation is required to reveal the role of plastic and elastic effects individually and mutually in establishing the flow field in a wide range of Bingham and Deborah numbers. This can be explored via a computational study since practical limitations exist in tackling this problem 550 experimentally. For example, it is not possible to change the Deborah number in our experiments independent of other parameters such as Bingham number. Also, it is not feasible to increase the Deborah number significantly with the aid of conventional yield stress fluids such as Carbopol gels.

Variation of disturbance velocity around one particle at fixed distances from the particle center ( $r$ fixed) is illustrated in Figs. 11a and 11b. It shows more clearly the fore-aft asymmetry in the Carbopol gel compared to that of the Newtonian fluid. Velocity is normalized with its maximum value at each distance, $u_{c, r}$ in Figs. $11 \mathrm{a}$ and $11 \mathrm{~b}$.

The disturbance field shows how regions around a particle are affected by the presence of a particle. When disturbance velocity is zero or very small at a region it means this region lies outside of the zone influenced by the particle. Studying the disturbance fields around one particle is thus essential to predict ${ }_{565}$ the interaction of two particles, and consequently, the bulk behavior of dilute suspensions. The extent of disturbance is better seen on the velocity profiles. 


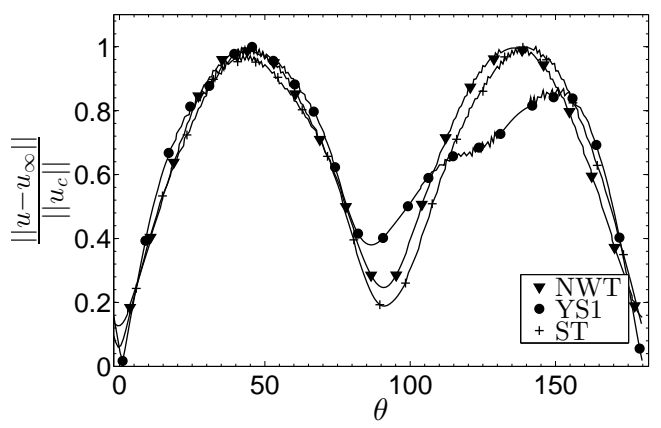

(a)

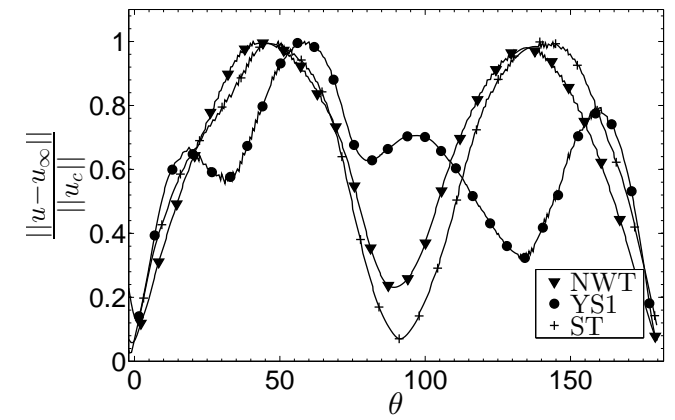

(b)

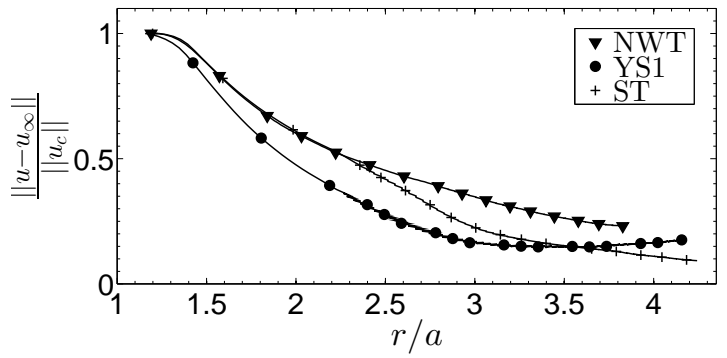

(c)

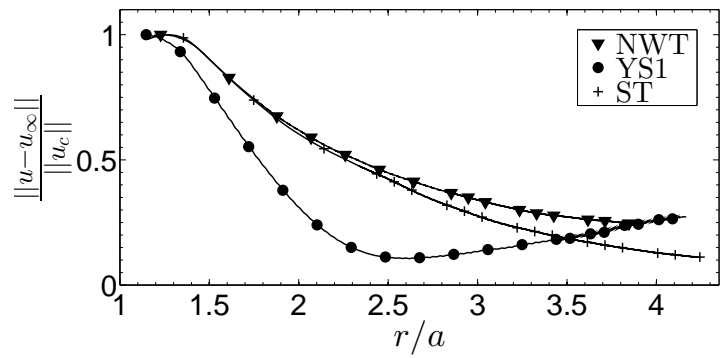

(d)

Figure 11: Variation of disturbance velocity at fixed distances ((a) $r / a=1.8$, (b) $r / a=2.3$ ) around one particle in different test fluids: NWT at $\dot{\gamma}=0.27 \mathrm{sec}^{-1}$, YS1 at $\dot{\gamma}=0.34 \mathrm{sec}^{-1}$ $(B, D e)=(1.23,0.15)$, and ST at $\dot{\gamma}=0.26 \mathrm{sec}^{-1} D e=1.03$. Variation of disturbance velocity along different directions in different test fluids: (c) $\theta=45^{\circ}$, (d) $\theta=135^{\circ}$. 
Figs. 11c and $11 \mathrm{~d}$ show the variation of disturbance velocity around one particle along different directions ( $\theta$ fixed) normalized with the maximum disturbance velocity along each direction, $u_{c, \theta}$. It is evident that the disturbance velocity decays more rapidly in the case of the yield stress fluid and shear thinning fluid. The maximum decay occurs in the flow of Carbopol gel around one particle. This means two particles will feel each other at a farther distance in a Newtonian fluid than in a generalized Newtonian fluid.

\subsection{Interaction of two particles in a linear shear flow}

In this section we study experimentally the interaction of two spherical PMMA particles in a linear shear flow of Newtonian, yield stress and shear thinning fluids. First, we compare our experimental results for the case of a Newtonain suspending fluid with the existing models [20] and analytical solutions 1 describing the relative motion of two particles in a linear shear flow without the inertia. We proceed afterwards to study the non-Newtonian effects on the interaction of particles in a linear shear flow.

\subsubsection{Interaction of two particles in a linear shear flow of a Newtonian fluid: theory and experiment}

Fig. 12 shows the schematic of a particle trajectory around a reference particle in a linear shear flow. Depending on the initial offset, $y_{0} / a$, the particles follow different trajectories. If the initial offset is small enough, two particles collide and separate further apart on the recession zone (symmetry is broken). However, if the initial offset is large enough that they do not make contact, the corresponding trajectory is expected to be symmetric due to the symmetry of the Stokes equations. It is noteworthy to mention that in the case of smooth particles with no surface roughness, a contact is not possible due to divergence of lubrication forces. However, practical contact occurs due to unavoidable 595 roughness at the surface of particles. For more details see theoretical [20, 1] and experimental works $92,19,22,15$.

The interaction of two particles could be described at different ranges of separation by accurate hydrodynamics functions based on works by Batchelor [1] and Da Cunha [20. It is assumed that inertial and Brownian effects are negligible, particles are neutrally buoyant and spherical. The appropriate set of hydrodynamic functions must be chosen according to the separation of two particles, $r$ and the roughness, $\varepsilon$. Using the aforementioned hydrodynamic functions we calculated the relative trajectories of two particles via 4th-order

605 Runge-Kutta to march in time. The results are plotted in Fig. 13a. The trajectories fall into two categories of asymmetric and symmetric whether or not a contact occurs respectively.

Here, we present our experimental results for two particles suspended in a linear shear flow of a Newtonian fluid. The experimental trajectory map of two 
a)
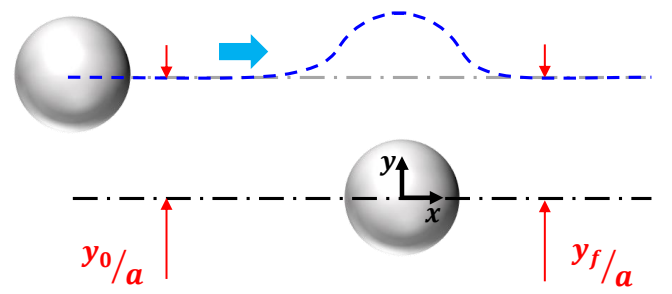

b)

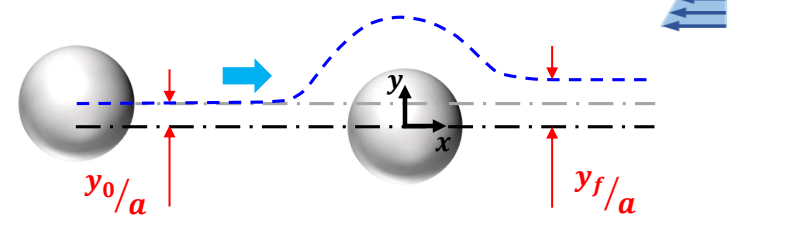

Figure 12: A schematic of two particles subjected to a shear flow and the general shapes of their trajectory: a) trajectory when two particles pass each other with no collision. b) trajectory when two particles collide.

particles is shown in Fig. 14. In addition, we have compared the experimental trajectory map with those calculated from theoretical solutions in Fig. 13b The best match is achieved by manually setting the roughness to $\varepsilon_{\text {theo }}=5.5 \times 10^{-4}$ in the model which is close to the peak value of roughness, $\varepsilon_{\exp }=6 \pm 3 \times 10^{-4}$ 615 reported by Phong in [21] for particles from the same batch. We see a great agreement between the theoretical and experimental trajectory map. The relative trajectories are symmetric with respect to y axis between the approach and the recession side if two particles do not contact. However, at lower initial offsets, when particles come into contact due to an unavoidable roughness at ${ }_{620}$ the particles surfaces, two particles separate further apart on their recession. Consequently, the particle trajectories are fore-aft asymmetric. It is evident that all trajectories along which the particles come into a contact will collapse on each other at the downstream after separation.

Particles are tracked via PTV and the flow field is investigated via PIV simultaneously. Therefore, we can link the particle trajectories to the information obtained from the flow field. Fig. 15 illustrates a typical example of a trajectory line with its corresponding velocity and local shear rate colormaps at different points along the trajectory line for two particles in a linear shear flow ${ }_{630}$ of a Newtonian fluid. The second particle approaches the reference particle from $x / a<0$. When particles are far from each other, the distribution of shear rate around them resembles that of a single particle, i.e., the particles do not see each other. The particles interact as they approach, and the shear rate distribution and velocity field around them change correspondingly. After they come into contact, they seem to get locked together and rotate like a single body (between 


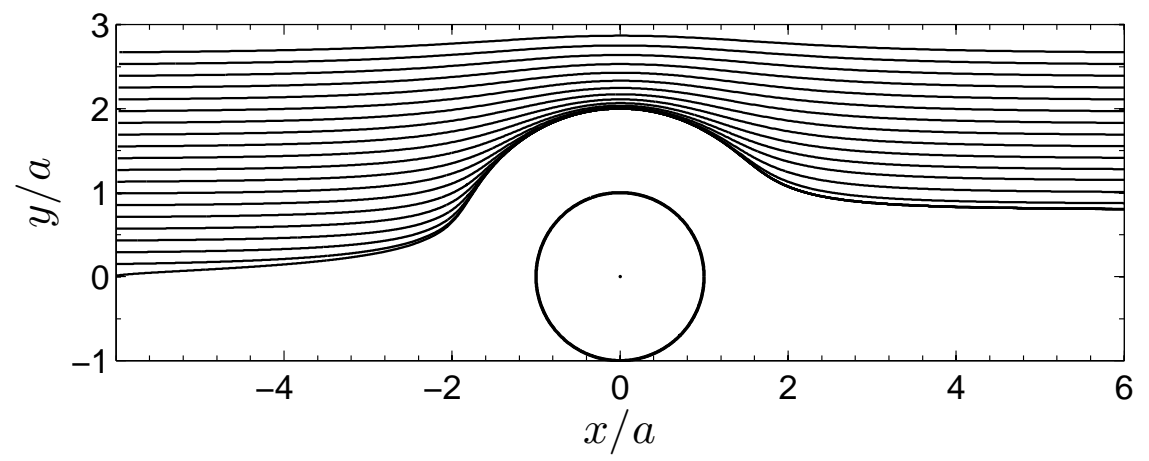

(a)

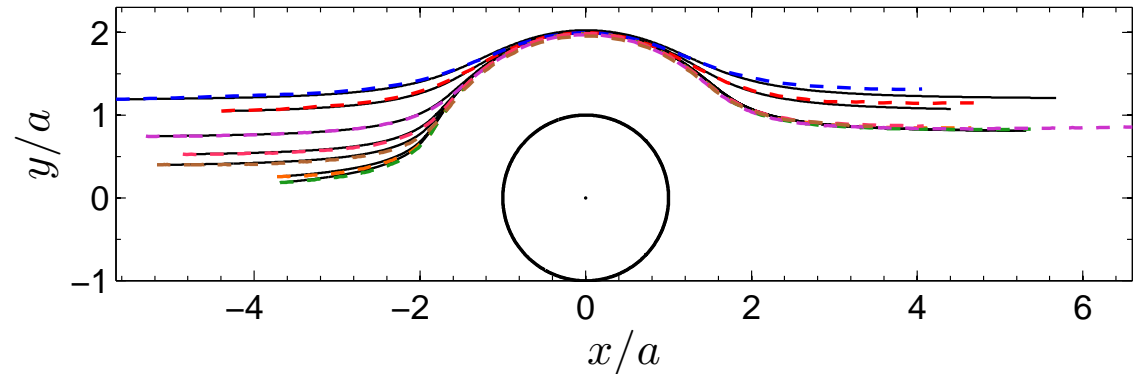

(b)

Figure 13: a) Relative trajectory map calculated via Da Cunha's model [20], $\varepsilon=5.5 \times 10^{-4}$. b) Relative trajectories obtained from the theoretical solution compared with those measured from the experiment (dashed colored lines) with the same initial offsets $(y / a$ wit $x<0)$.

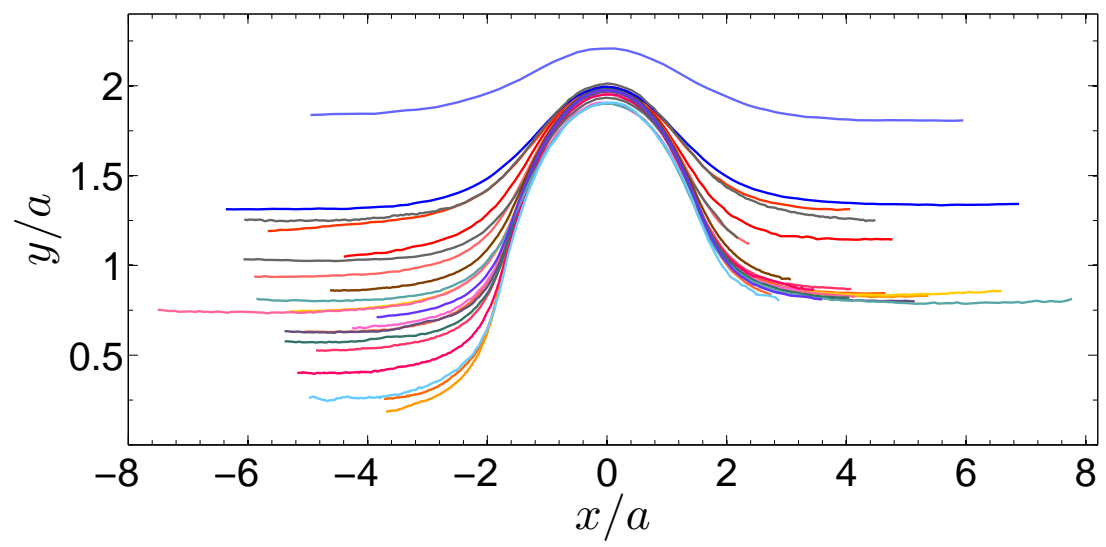

Figure 14: Trajectory map of two particles in a linear shear flow of the Newtonian fluid. The reference particle is located at the origin and the second particle is initially at $x / a<0$. 
points B and D in Fig. 15p then separate from each other. Shear rate fields are normalized by the far-field shear rate.

\subsubsection{Interaction of two particles in a linear shear flow of a yield stress fluid: experiment}

In this section we present our experimental results on the interaction of two PMMA spherical particles in a linear shear flow of Carbopol gel, which is a yield stress fluid (see Sections 2.3.2 and 2.4). In such case, a theoretical solution does not exist due to the nonlinearity of the governing equations of motion, even in the absence of inertia. While the majority of the experimental works and

${ }_{645}$ simulations focused on the settling of particles in yield stress fluids, there are no simulation or experimental work on the interaction of two particles in a linear shear flow of a yield stress fluid in the literature. However, a paper relating a numerical 2D study of the interaction of pairs of particles in an ideal Bingham fluid is under review at the same time as our paper; our experimental results will be qualitatively compared to the simulation results when relevant 93 .

In the absence of inertia, the knowledge of roughness and initial offset are sufficient to predict the interaction, and consequently, the relative trajectory of two particles when we are dealing with Newtonian fluids. However, there are ${ }_{655}$ more parameters influencing the interactions of two particles in a yield stress fluid. We expect that the value of Bingham number should strongly affect the relative motion of two particles.

Moreover, viscoelastic effects are not always negligible when dealing with non-ideal yield stress fluids and their contribution must be evaluated (see [44, 45]). According to the range of Deborah number in our experiments, De $\epsilon$ $[0.04,1.3]$ we believe that viscoelastic effects can play an important role, which is consistent with 44 .

665 In addition, shear history is another parameter which affects the interaction of two particles due to the strain hardening in the non-ideal yield stress test fluids. As discussed earlier in sec. 2.4 for a sample of Carbopol gel, the material undergoes different transient flow states depending on the applied shear history. Our results show that when the material is pre-sheared in a negative direction, ${ }_{670}$ the trajectories experience a relatively longer transient regime (results not included). This is consistent with our results in Fig. 3 which suggest that the material reaches a steady state at larger strains under negative pre-shear. In the course of this study, we apply the same shear history in all of the experiments via adopting the positive pre-shear procedure in order to avoid strain hardening and to be as close as possible to a model plastic behavior. However, we should mention that the dimension of our Couette-cell is large enough to allow us to apply sufficient amounts of pre-strain to reach steady state condition, regardless of the shear history. 


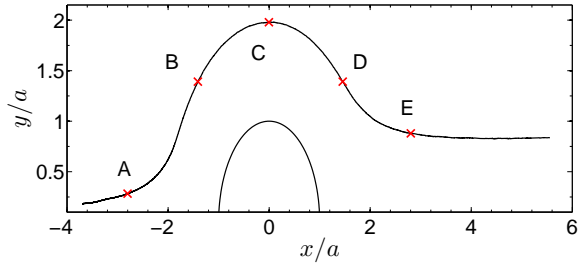

(a)

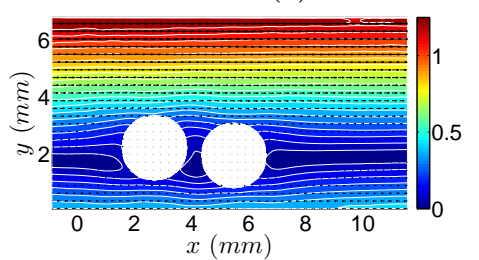

(b)

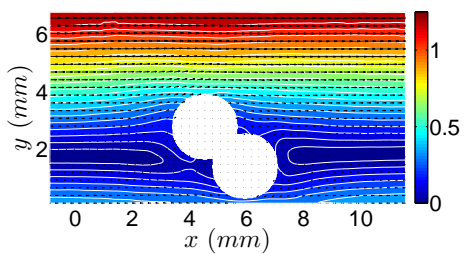

(d)

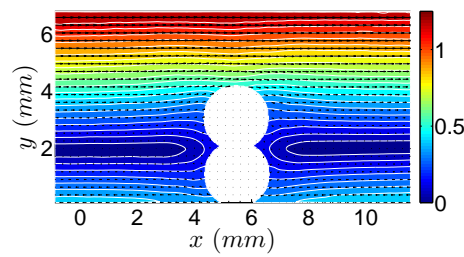

(f)

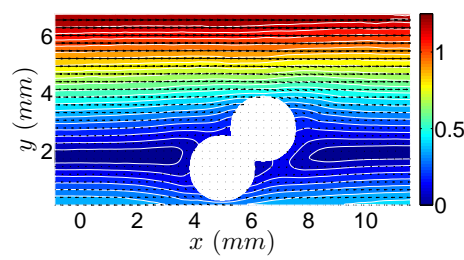

(h)

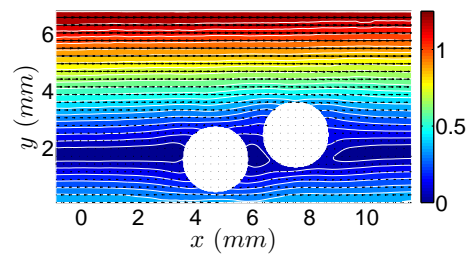

(j)

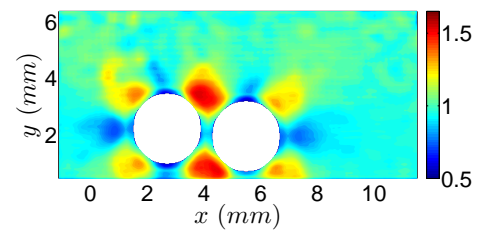

(c)

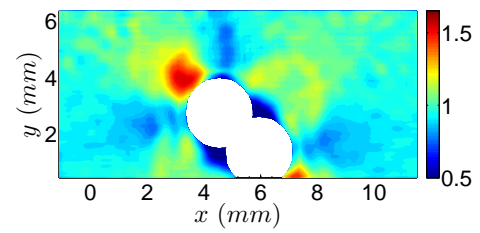

(e)

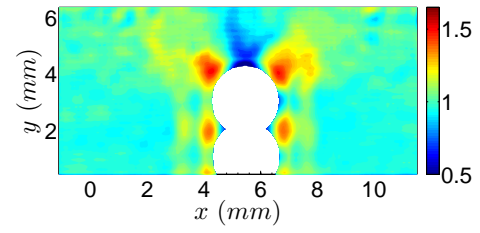

(g)

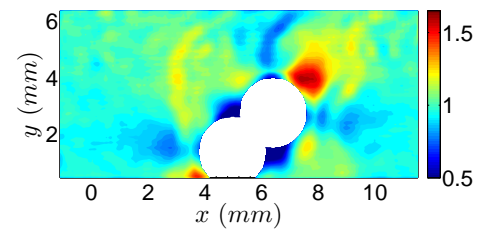

(i)

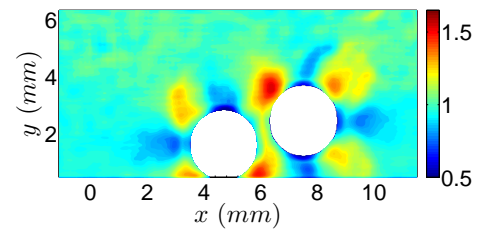

(k)

Figure 15: (a) Trajectory line of two particles in the Newtonian fluid subjected to a shear rate of $\dot{\gamma}=.27 \mathrm{sec}^{-1}$. (b-k) Left column is the velocity fields at different points marked along the trajectory line (A to $\mathrm{E}$ ) while the right column is the corresponding normalized shear rate fields. 


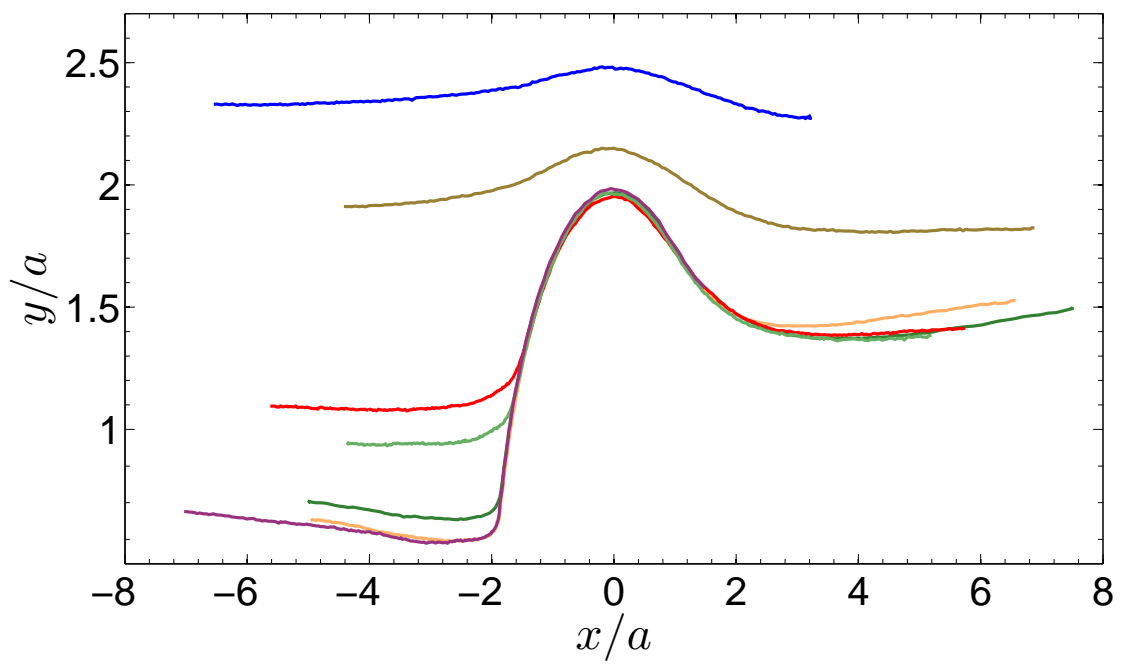

Figure 16: Trajectory map of two particles in a shear flow of the Carbopol gel YS1 at $\dot{\gamma}=0.34$ $\sec ^{-1}(B, D e)=(1.23,0.15)$.

While shearing the material we study the interaction of particles and the flow field via performing PTV and PIV respectively. Fig. 16 shows the trajectory map of particles in a Carbopol gel at $\dot{\gamma}=0.34 \mathrm{sec}^{-1}, B=1.23$ and $D e=0.15$. Two features are evident. First, the fore-aft asymmetries exist for all the trajectories including those with no collisions of particles. When the initial offset is large enough that there is no contact, particles experience a negative drift along the $y$-direction after passing each other (i.e. $y_{f}-y_{0}<0$ ). We think that this pattern can be attributed to the elasticity of the test fluid since no such behavior is observed in simulations when the fluid is considered ideal visco-plastic (e.g. Bingham model) 93. Second, for trajectories with small initial offsets, 690 the second particle moves downward along the velocity gradient direction on the approach side while it moves upward on the recession side. The same pattern is observed in the simulations by Fahs et al. in 93 for yield stress fluids as well as Newtonian fluids. These local minima in trajectories disappeared in their results for the Newtonian fluid when the domain size is increased from $24 a \times 12 a$ to $96 a \times 48 a$. However, this pattern for the yield stress fluid (with $B=10$ ) disappeared at a larger domain size, $192 a \times 96 a$. Hence, we can conclude that this might be due to the interplay of wall effects and non-Newtonian behavior.

Fig. 17 shows trajectories of two particles in a Carbopol gel at two different Bingham numbers, starting from approximately equal initial offsets. As expected, the particle trajectories strongly depend on the Bingham number. As we increase the Bingham number the second particle approaches the reference particle to a close distance and separates with a larger upward drift. This can be related to the stronger decay of the disturbance velocity at larger Bingham values around a single particle (see Sec. 3.2 . This feature, which has been also 


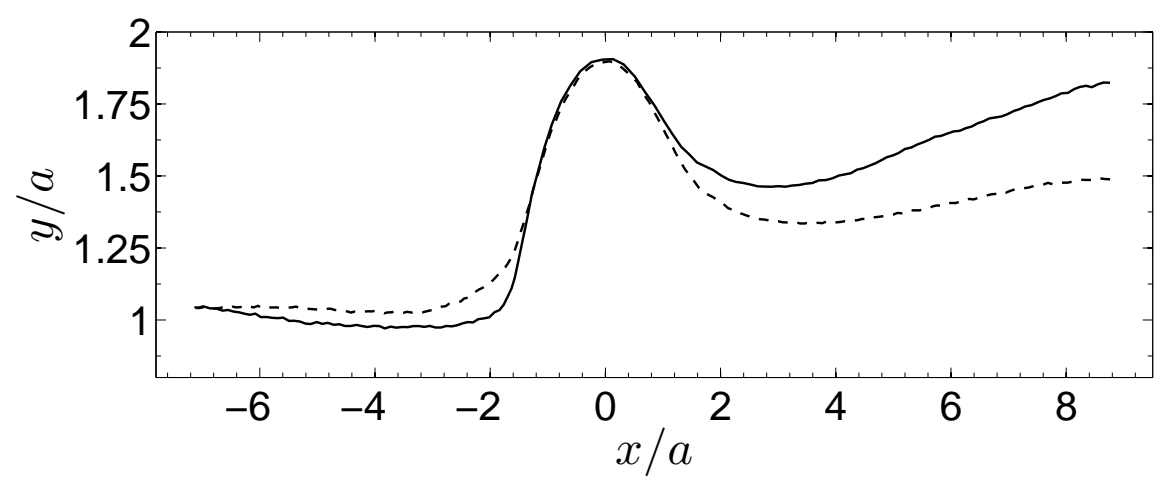

Figure 17: Relative trajectories of two particles in the Carbopol gel YS1 with similar initial offsets at two different Bingham numbers. Dashed line corresponds to $\dot{\gamma}=1.70 \sec ^{-1}(B, D e)=$ $(0.55,0.36)$ while the solid line represents $\dot{\gamma}=0.21 \mathrm{sec}^{-1}(B, D e)=(1.57,0.12)$.

observed in simulations of Fahs et al. [93, implies larger asymmetry in the PDF, and consequently, larger normal stress differences in the yield stress suspensions as we increase the Bingham number.

Fig. 18 shows a typical example of a trajectory line with its corresponding velocity and local shear rate colormaps at different points along the trajectory line for two particles in a linear shear flow of a yield stress fluid. Shear rate fields are normalized with the applied shear rate at the belt. The second particle approaches the reference particle from $x / a<0$. We see that particles interact as they approach and the shear rate distribution and velocity field around them change (see colormpas associated with point A, Figs. $18 \mathrm{~b}$ and c). After they come into contact they seem to get locked together and rotate like a single body (between points B and C in Fig. 18). They separate from each other afterwards on their recession.

\subsubsection{Interaction of two particles in a linear shear flow of a shear thinning} fluid: experiment

A Carbopol gel exhibits both yield stress and shear thinning effects. In order to investigate the effect of each non-Newtonian behavior individually, we perform similar experiments with a shear thinning test fluid without a yield stress. We use a Hydroproxypyl Guar solution which is transparent with negligible thixotropy at low concentrations (see Sections 2.3 and 2.4).

A map of the relative trajectory map of two particles in a linear shear flow of the guar gum solution, ST, is illustrated in Fig. 19. Unlike yield stress sus730 pending fluids, trajectories do not exhibit downward and upward motions at the approach and recession zone respectively. A slight asymmetry exists when particles do not come into a contact, but this is much smaller than that of yield stress suspending fluids. When a contact occurs, the trajectories are all asym- 


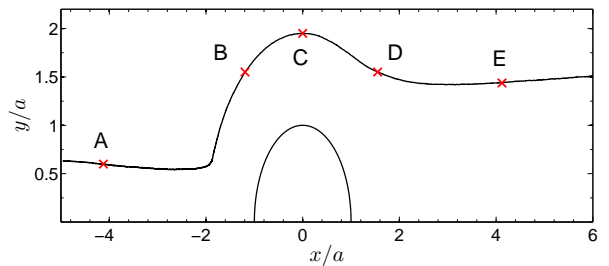

(a)

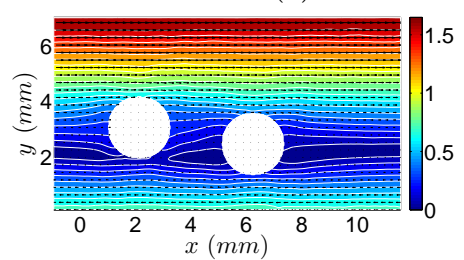

(b)

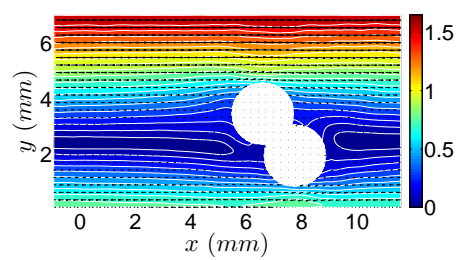

(d)

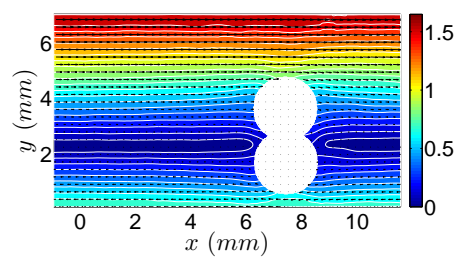

(f)

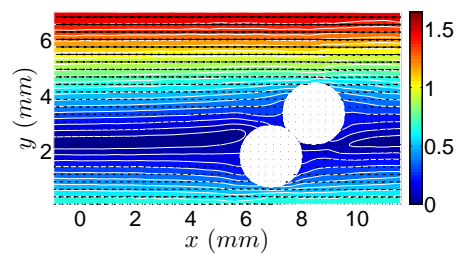

(h)

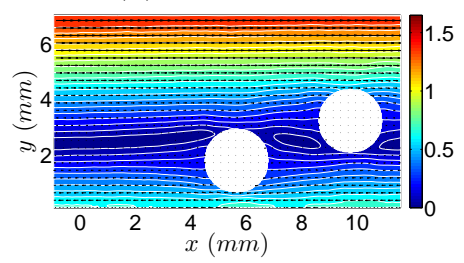

(j)

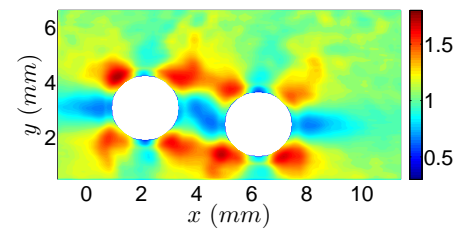

(c)

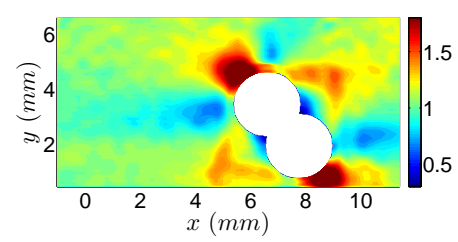

(e)

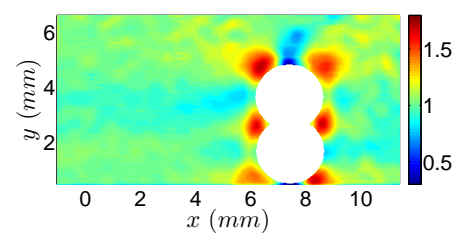

(g)

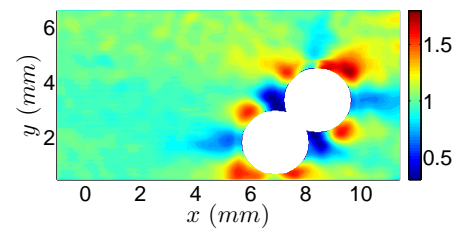

(i)

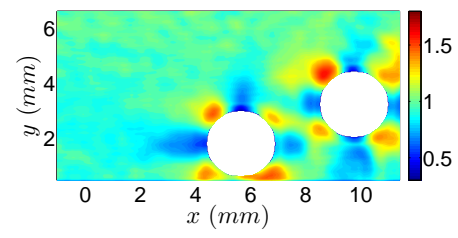

(k)

Figure 18: (a) Trajectory line of two particles in the Carbopol gel, YS1 at $\dot{\gamma}=0.34 \mathrm{sec}^{-1}$ $(B, D e)=(1.23,0.15)$. (b-k) Left column is the velocity fields at different points marked on the trajectory line (A to $\mathrm{E}$ ) while the right column is the corresponding normalized shear rate fields. 


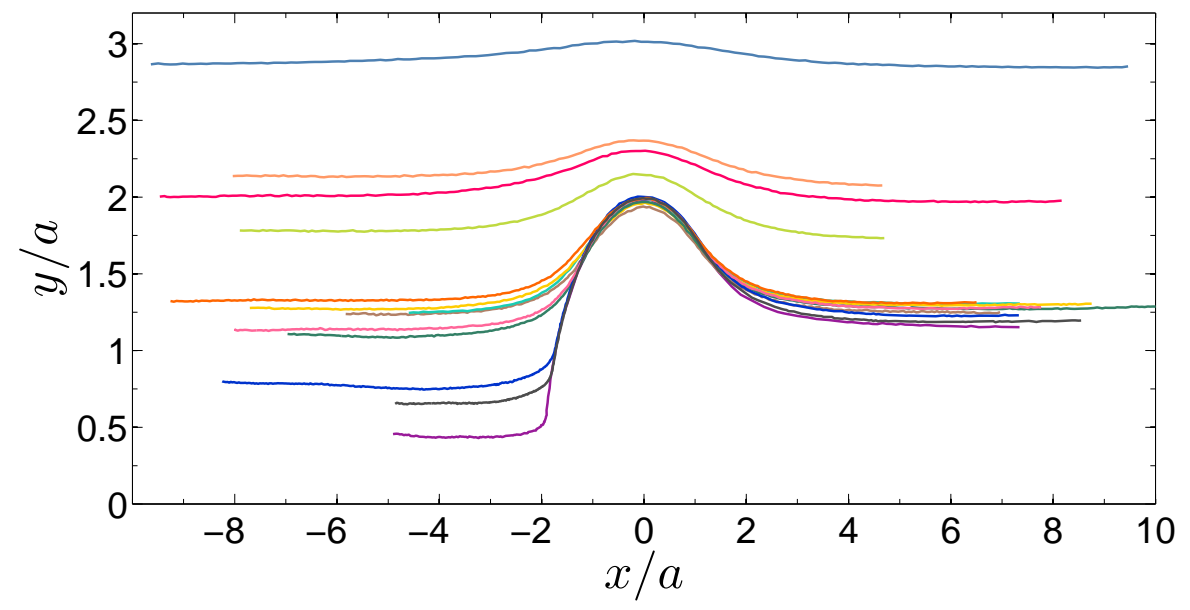

Figure 19: Trajectory map of two particles subjected to a shear flow of ST at $(\dot{\gamma}, D e)=(0.26$ $\left.\sec ^{-1}, 1.03\right)$.

metric.

735

Fig. 20 illustrates a sample trajectory with its corresponding velocity and shear rate fields at different points along the trajectory line for two particles in the guar gum solution ST (see Table 1). The second particle approaches the reference particle from $x / a<0$. Shear rate fields are normalized with the

\subsubsection{Particle trajectories versus streamlines}

As mentioned earlier in Section 3.2.2, the disturbance velocity decays more rapidly in the non-Newtonian fluids considered in this study. In other words, the influence zone around a single particle is smaller when dealing with yield stress and shear thinning fluids compared to the Newtonian fluid. In Fig. 21 we compared the trajectories of two particles subjected to a shear flow with the streamlines around a single particle (experimental velocity field). We can see that they overlap up to closer distances in the Carbopol gel and guar gum solution.

750

The streamlines around one particle can be viewed as the limiting form of when two particles are far away or when one particle is much smaller than the other one. The discrepancy between the fluid element streamlines and trajectories is related to the lubrication and contact of the particles. Fig. 21 shows pairwise interaction does not occur. Further computational and theoretical investigations are needed to build up trajectory maps of particle pairs in complex fluids from the flow field around a single particle in shear flows of complex fluids. 


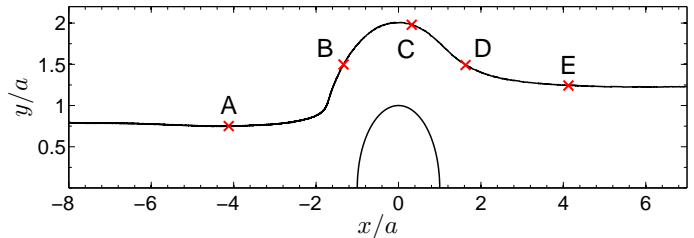

(a)

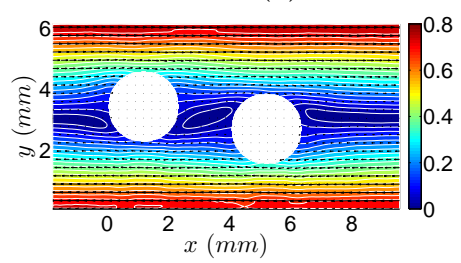

(b)

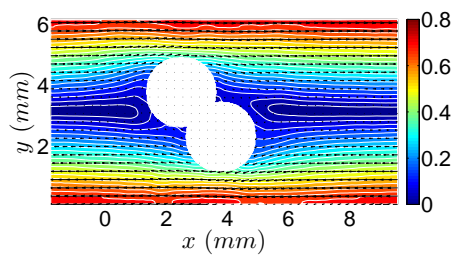

(d)

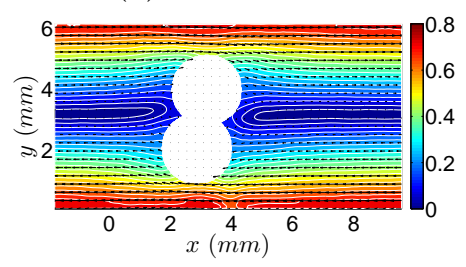

(f)

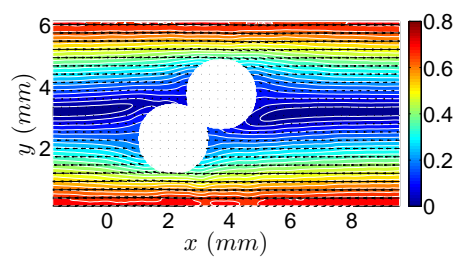

(h)

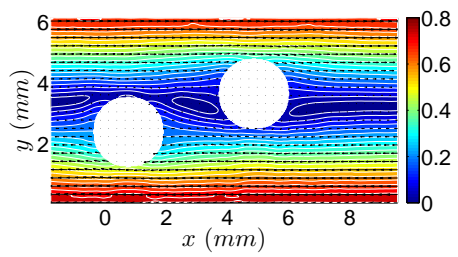

(j)

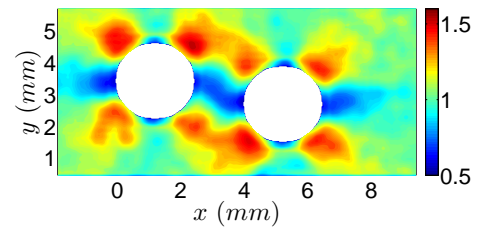

(c)

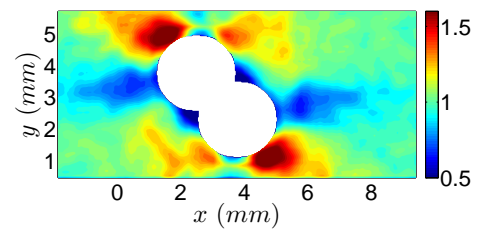

(e)

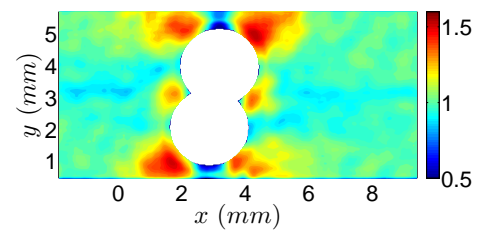

(g)

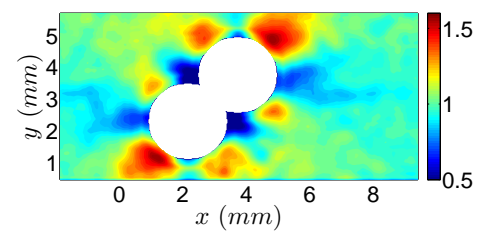

(i)

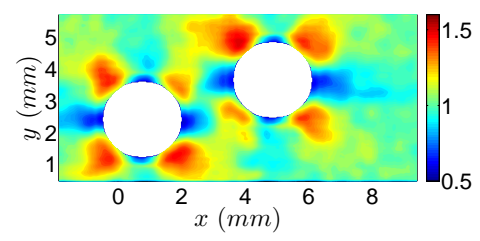

(k)

Figure 20: (a) Trajectory line of two particles in the guar gum solution, ST at $\dot{\gamma}=0.26 \mathrm{sec}^{-1}$ $D e=1.03$. (b-k) Left column is the velocity fields at different points marked on the trajectory line (A to $\mathrm{E}$ ) while the right column is the corresponding normalized shear rate fields. 


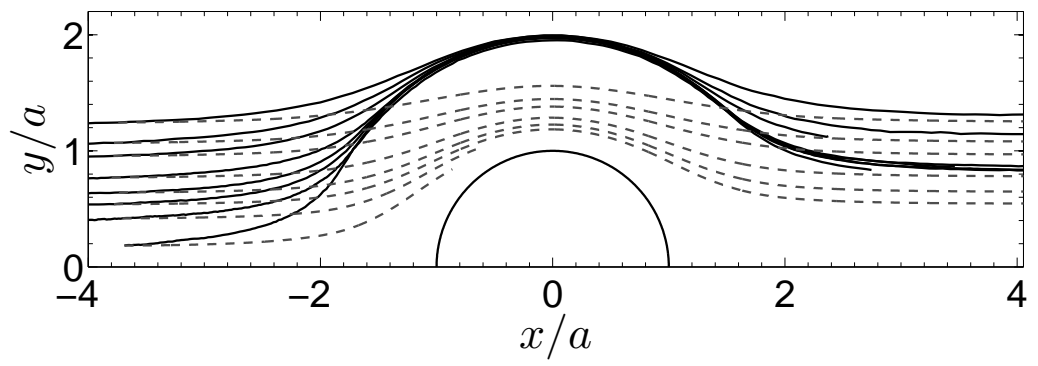

(a)

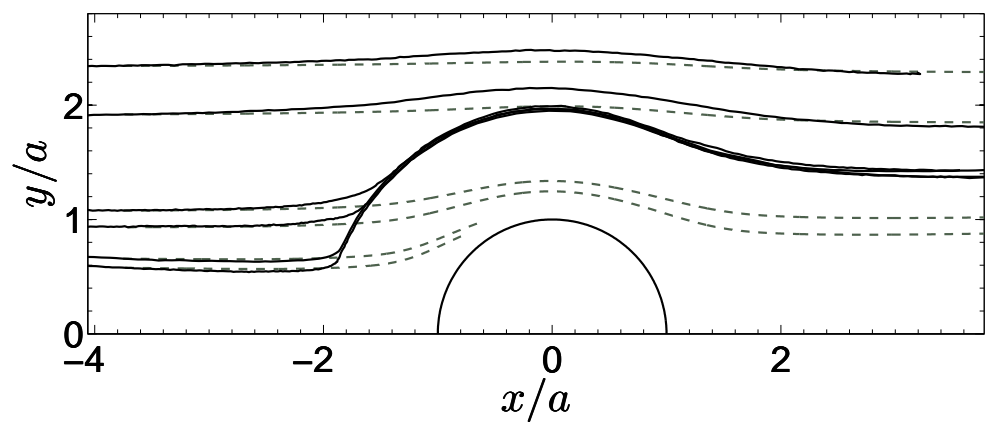

(b)

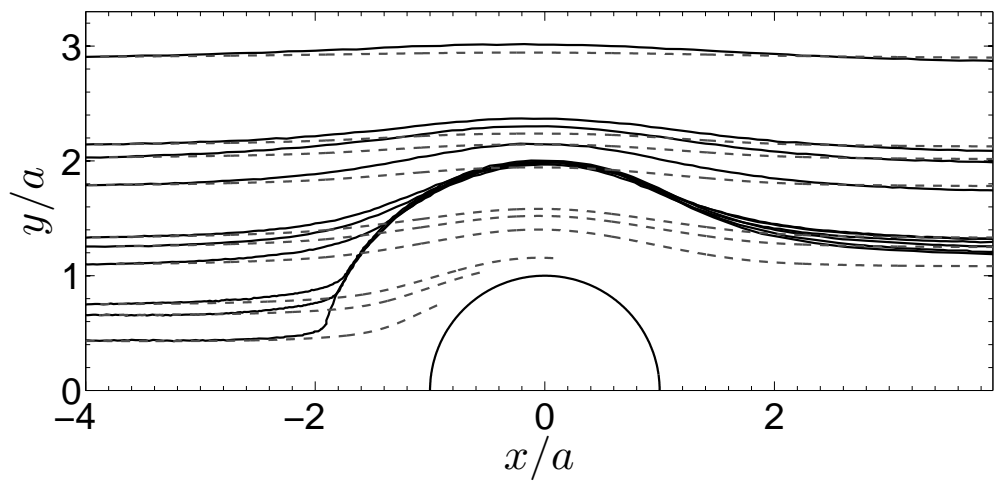

(c)

Figure 21: Two-particle trajectories (solid lines) compared with the streamlines around one particle (dashed lines) in shear flows of different fluids: (a) NWT at $\dot{\gamma}=0.27 \mathrm{sec}^{-1}$, (b) YS1 at $\dot{\gamma}=0.34 \mathrm{sec}^{-1}(B, D e)=(1.23,0.15)$ and (c) ST at $\dot{\gamma}=0.26 \mathrm{sec}^{-1} \mathrm{De}=1.03$. 


\section{Discussion and conclusions}

In this work, we have developed an accurate experimental technique to study the interaction of two spherical particles in linear shear flows of Newtonian, yield stress and shear thinning fluids. We have made use of PIV and PTV techniques to measure the velocity fields and particle trajectories respectively. Rheometry is employed in order to characterize the behavior of our test fluids.

We showed in Section 3.1 that we can establish a linear velocity profile in our Newtonian and non-Newtonian test fluids. In addition, for yield stress fluids, we observed that stress inhomogeneity (naturally present due to any imperfection in the set-up or the test fluid) could project to a larger amount of shear rate inhomogeneity as we increase the Bingham number. By restricting the range of Bingham number to $B<2$, we managed to eliminate this effect and achieve a linear shear flow in Couette device.

Next, we studied the flow around one particle when it is subjected to a linear shear flow. Our results are in a very close agreement with the theoretical solution for a Newtonian suspending fluid. Also the length scale of variation of the disturbance velocity is significantly smaller in yield stress fluids compared to that of Newtonian fluids. This affects the interaction of two particles, and consequently, the bulk rheology of suspensions of noncolloidal particles in shear thinning and yield stress fluids.

We provided the first direct experimental measurement of the flow disturbance around a sphere in a yield stress fluid. This can serve as a benchmark 785 for simulations when dealing with suspensions of noncolloidal particles in yield stress fluids. Our study shows that Carbopol gel exhibits significant viscoelastic behavior which affects the particle interactions. We observed that even the disturbance field around a single particle in a shear flow cannot be explained without considering the viscoelastic effects. Hence, employing elastoviscoplastic (EVP) constitutive models [46 47] are necessary when accurate simulations are considered 44. Due to the experimental limits, further theoretical and computational studies are required to characterize the contribution of elastic and plastic effects in establishing the flow field around a single particle.

In the next step, we studied the interaction of a pair of neutrally buoyant particles in linear shear flows of Newtonian, yield stress and shear thinning fluids. In the case of Newtonian suspending fluids, we observed a very close agreement between our measurements and the available theoretical solution, which shows the merit of our experimental method. Subsequently, the same method has been employed to study the problem with yield stress and shear thinning suspending fluids which we have no theoretical solutions available for. As it is evident in Fig. 22, fore-aft asymmetry is enhanced for trajectories of particles in yield stress fluids (also observed in simulations of Fahs et al. 93]) and shear thinning fluids. Even a slight asymmetry has been observed in tra- 
jectories with no collision. These observations imply greater asymmetry in the PDF and stronger normal stress differences in the yield stress suspensions.

It is noteworthy to mention that for yield stress suspending fluids, in the absence of inertia, the interaction of particles depend on various parameters such ${ }_{810}$ as Bingham number, Deborah number, shear history, initial offset and roughness. Hence, obtaining the entire trajectory space is not feasible experimentally for yield-stress fluids. However, overall trends and patterns could be understood by investigating a limited number of systematic measurements. The effect of different parameters on the interaction of particles is investigated in this study.

815

As mentioned in Section 3.2.2, in the guar gum solution and Carbopol gel, variations along the trajectory lines are confined in a closer neighborhood of the particle. We can link this observation to the variation of the disturbance velocity field around one particle in yield stress fluids where the length scale of the decay is smaller than that in Newtonian suspending fluids (see Figs. 9 , 11c and 11d ). This feature has been observed in the numerical simulations of Fahs et al. 93. It means that two particles feel each other's presence at closer distances, and when they do, the interactions are more severe. One can conclude that the short-range interactions are more important when dealing with

825 yield stress suspending fluids. Due to the limited resolution of the experimental measurements close to the particles, especially when they are touching or very close (separations of the order the size of the interrogation window), accurate simulations with realistic constitutive models are required to understand and characterize the short-range hydrodynamic interactions, particularly the lubrication forces.

Another distinct feature observed during the motion of two particles in a yield stress fluid is the downward and upward motion of the second particle along the velocity gradient direction during approach and recession. This phe835 nomenon could affect the microstructure, and consequently, the PDF of yield stress suspensions. This pattern has been observed experimentally for shear thinning suspending fluids in 94. Also, similar behavior is observed for both Newtonian and yield stress fluids in the simulations of Fahs et al. 93. By increasing the gap size, $w / a$, the downward and upward motion disappeared in 840 their results for Newtonian fluid. However, for yield stress fluid, such behavior disappeared at larger gap sizes. We have not observed this feature during the motion of two particles in the shear thinning fluid in the course of this project, but it is perhaps due to the fact that this behavior is present only at initial offsets smaller than the range covered in our experiments. The confinement 845 effects might be responsible for this behavior. The extent of such effects could be amplified in the presence of yield stress fluids. Further investigations are needed to understand the underlying mechanisms properly. 


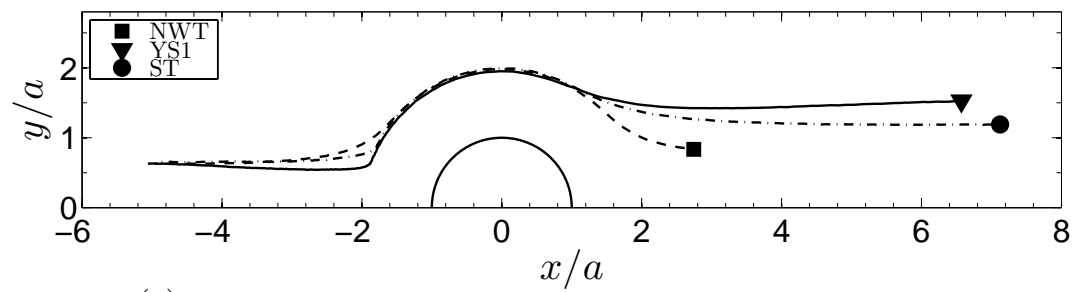

(a)

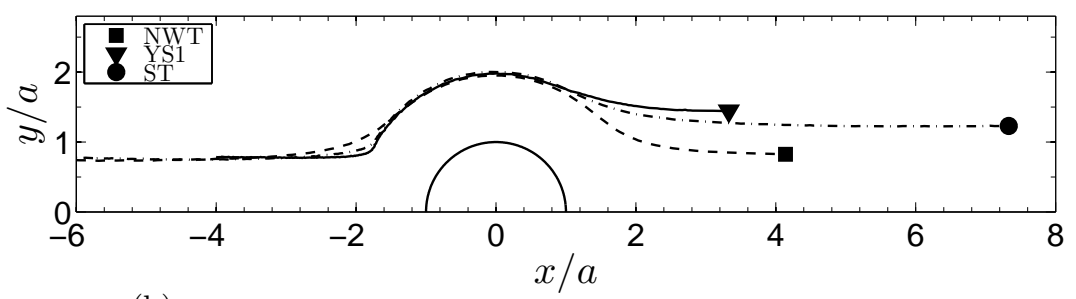

(b)

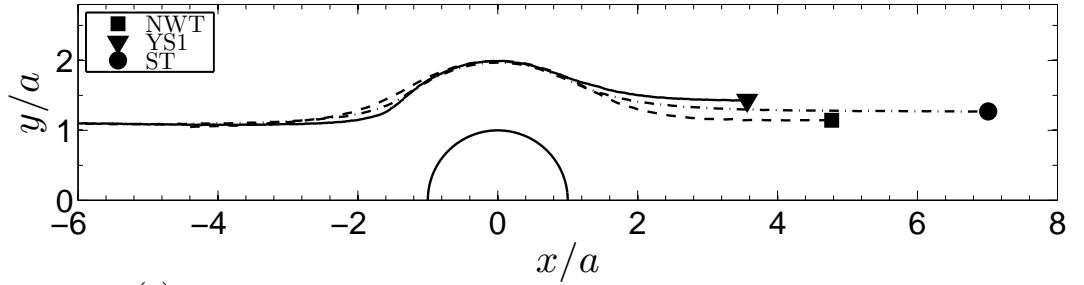

(c)

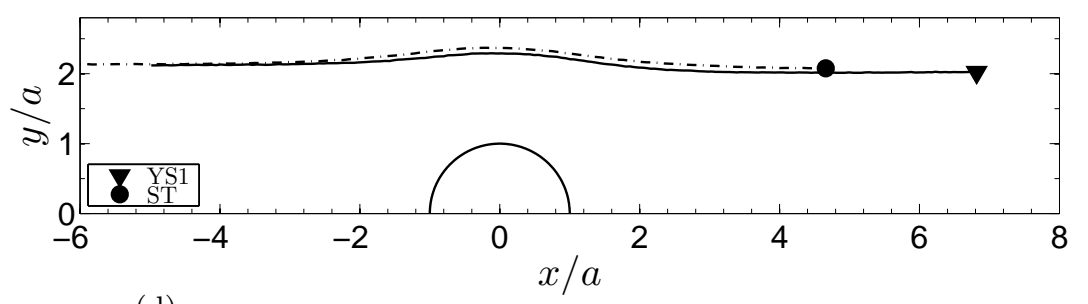

(d)

Figure 22: a-d) Relative trajectories of two particles in shear flows of different test fluids with similar initial offsets: $y_{0} / a=0.63$ (a), 0.75 (b), 1.05 (c), 2.12 (d). Test fluids include NWT at $\dot{\gamma}=0.27 \mathrm{sec}^{-1}, \mathrm{YS} 1$ at $\dot{\gamma}=0.34 \mathrm{sec}^{-1}(B, D e)=(1.23,0.15)$ and ST at $\dot{\gamma}=0.26 \mathrm{sec}^{-1}$ $D e=1.03$. 


\section{Acknowledgments}

850 This research was supported by National Science Foundation (Grant No. CBET-1554044-CAREER) via the research award (S.H.).

\section{References}

[1] G. Batchelor, J.-T. Green, The hydrodynamic interaction of two small freely-moving spheres in a linear flow field, Journal of Fluid Mechanics $56(02)(1972) 375-400$.

[2] G. Batchelor, J. Green, The determination of the bulk stress in a suspension of spherical particles to order c 2, Journal of Fluid Mechanics 56 (03) (1972) 401-427.

[3] D. Jeffrey, Y. Onishi, Calculation of the resistance and mobility functions for two unequal rigid spheres in low-reynolds-number flow, Journal of Fluid Mechanics 139 (1984) 261-290.

[4] S. Kim, R. T. Mifflin, The resistance and mobility functions of two equal spheres in low-reynolds-number flow, The Physics of fluids 28 (7) (1985) 2033-2045.

[5] D. Jeffrey, The calculation of the low reynolds number resistance functions for two unequal spheres, Physics of Fluids A: Fluid Dynamics 4 (1) (1992) $16-29$.

[6] S. Kim, S. J. Karrila, Microhydrodynamics: principles and selected applications, Courier Corporation, 2013.

[7] G. Batchelor, The effect of brownian motion on the bulk stress in a suspension of spherical particles, Journal of fluid mechanics 83 (01) (1977) $97-117$.

[8] J. F. Brady, J. F. Morris, Microstructure of strongly sheared suspensions and its impact on rheology and diffusion, Journal of Fluid Mechanics 348 (1997) 103-139.

[9] I. E. Zarraga, D. T. Leighton Jr, Normal stress and diffusion in a dilute suspension of hard spheres undergoing simple shear, Physics of Fluids 13 (3) (2001) 565-577.

${ }_{880}$ [10] J. F. Brady, G. Bossis, Stokesian dynamics, Annual review of fluid mechanics 20 (1) (1988) 111-157.

[11] H. Brenner, M. E. O'Neill, On the stokes resistance of multiparticle systems in a linear shear field, Chemical Engineering Science 27 (7) (1972) 14211439. 
[12] S. Wakiya, C. Darabaner, S. Mason, Particle motions in sheared suspensions xxi: Interactions of rigid spheres (theoretical), Rheologica Acta 6 (3) (1967) 264-273.

[13] C. Lin, K. Lee, N. Sather, Slow motion of two spheres in a shear field, Journal of Fluid Mechanics 43 (01) (1970) 35-47.

[14] E. Guazzelli, J. F. Morris, A physical introduction to suspension dynamics, Vol. 45, Cambridge University Press, 2011.

[15] F. Blanc, F. Peters, E. Lemaire, Experimental signature of the pair trajectories of rough spheres in the shear-induced microstructure in noncolloidal suspensions, Physical review letters 107 (20) (2011) 208302.

[16] F. Blanc, E. Lemaire, A. Meunier, F. Peters, Microstructure in sheared non-brownian concentrated suspensions, Journal of rheology 57 (1) (2013) 273-292.

[17] F. Parsi, F. Gadala-Maria, Fore-and-aft asymmetry in a concentrated suspension of solid spheres, Journal of Rheology 31 (8) (1987) 725-732.

[18] C. Gao, S. Kulkarni, J. Morris, J. Gilchrist, Direct investigation of anisotropic suspension structure in pressure-driven flow, Physical Review E 81 (4) (2010) 041403.

[19] F. Blanc, F. Peters, E. Lemaire, Kinetics of owing dispersions. 9. doublets of rigid spheres (experimental), Journal of Colloid Interface Science 61 (1977) 44 .

[20] F. Da Cunha, E. Hinch, Shear-induced dispersion in a dilute suspension of rough spheres, Journal of Fluid Mechanics 309 (1996) 211-223.

[21] P. Pham, B. Metzger, J. E. Butler, Particle dispersion in sheared suspensions: Crucial role of solid-solid contacts, Physics of Fluids 27 (5) (2015) 051701.

[22] I. Rampall, J. R. Smart, D. T. Leighton, The influence of surface roughness on the particle-pair distribution function of dilute suspensions of noncolloidal spheres in simple shear flow, Journal of Fluid Mechanics 339 (1997) $1-24$.

[23] J. F. Morris, A review of microstructure in concentrated suspensions and its implications for rheology and bulk flow, Rheologica acta 48 (8) (2009) 909-923.

[24] A. Singh, P. R. Nott, Normal stresses and microstructure in bounded sheared suspensions via stokesian dynamics simulations, Journal of Fluid Mechanics 412 (2000) 279-301.

[25] A. Sierou, J. Brady, Rheology and microstructure in concentrated noncolloidal suspensions, Journal of Rheology 46 (5) (2002) 1031-1056. 
[26] J. J. Stickel, R. L. Powell, Fluid mechanics and rheology of dense suspensions, Annu. Rev. Fluid Mech. 37 (2005) 129-149.

[27] N. Phan-Thien, X.-J. Fan, B. C. Khoo, A new constitutive model for monodispersed suspensions of spheres at high concentrations, Rheologica acta 38 (4) (1999) 297-304.

[28] J. J. Stickel, R. J. Phillips, R. L. Powell, A constitutive model for microstructure and total stress in particulate suspensions, Journal of Rheology 50 (4) (2006) 379-413.

[29] J. J. Stickel, R. J. Phillips, R. L. Powell, Application of a constitutive model for particulate suspensions: Time-dependent viscometric flows, Journal of Rheology 51 (6) (2007) 1271-1302.

[30] R. M. Miller, J. P. Singh, J. F. Morris, Suspension flow modeling for general geometries, Chemical Engineering Science 64 (22) (2009) 4597-4610.

[31] J. F. Morris, F. Boulay, Curvilinear flows of noncolloidal suspensions: The role of normal stresses, Journal of rheology 43 (5) (1999) 1213-1237.

[32] J. Morris, J. Brady, Pressure-driven flow of a suspension: Buoyancy effects, International journal of multiphase flow 24 (1) (1998) 105-130.

[33] P. R. Nott, J. F. Brady, Pressure-driven flow of suspensions: simulation and theory, Journal of Fluid Mechanics 275 (1994) 157-199.

[34] R. R. Huilgol, N. Phan-Thien, Fluid mechanics of viscoplasticity, Springer, 2015.

[35] A. Putz, I. Frigaard, Creeping flow around particles in a bingham fluid, Journal of Non-Newtonian Fluid Mechanics 165 (5) (2010) 263-280.

[36] A. Beris, J. Tsamopoulos, R. Armstrong, R. Brown, Creeping motion of a sphere through a bingham plastic, Journal of Fluid Mechanics 158 (1985) 219-244.

[37] B. T. Liu, S. J. Muller, M. M. Denn, Convergence of a regularization method for creeping flow of a bingham material about a rigid sphere, Journal of non-newtonian fluid mechanics 102 (2) (2002) 179-191.

[38] J. Blackery, E. Mitsoulis, Creeping motion of a sphere in tubes filled with a bingham plastic material, Journal of non-newtonian fluid mechanics 70 (1) (1997) 59-77.

[39] M. Beaulne, E. Mitsoulis, Creeping motion of a sphere in tubes filled with herschel-bulkley fluids, Journal of non-newtonian fluid mechanics 72 (1) (1997) 55-71.

[40] B. Deglo de Besses, A. Magnin, P. Jay, Sphere drag in a viscoplastic fluid, AIChE journal 50 (10) (2004) 2627-2629. 
[41] A. Putz, T. Burghelea, I. Frigaard, D. Martinez, Settling of an isolated spherical particle in a yield stress shear thinning fluid, Physics of Fluids 20 (3) (2008) 033102.

[42] Y. Holenberg, O. Lavrenteva, A. Liberzon, U. Shavit, A. Nir, Ptv and piv study of the motion of viscous drops in yield stress material, Journal of Non-Newtonian Fluid Mechanics 193 (2013) 129-143.

[43] B. Gueslin, L. Talini, B. Herzhaft, Y. Peysson, C. Allain, Flow induced by a sphere settling in an aging yield-stress fluid, Physics of Fluids 18 (10) (2006) 103101.

[44] D. Fraggedakis, Y. Dimakopoulos, J. Tsamopoulos, Yielding the yieldstress analysis: a study focused on the effects of elasticity on the settling of a single spherical particle in simple yield-stress fluids, Soft matter 12 (24) (2016) 5378-5401.

[45] D. Fraggedakis, Y. Dimakopoulos, J. Tsamopoulos, Yielding the yield stress analysis: A thorough comparison of recently proposed elasto-visco-plastic (evp) fluid models, Journal of Non-Newtonian Fluid Mechanics 238 (2016) 170-188.

[46] P. Saramito, A new constitutive equation for elastoviscoplastic fluid flows, Journal of Non-Newtonian Fluid Mechanics 145 (1) (2007) 1-14.

[47] P. Saramito, A new elastoviscoplastic model based on the herschel-bulkley viscoplastic model, Journal of Non-Newtonian Fluid Mechanics 158 (1) (2009) 154-161.

[48] C. J. Dimitriou, R. H. Ewoldt, G. H. McKinley, Describing and prescribing the constitutive response of yield stress fluids using large amplitude oscillatory shear stress (laostress), Journal of Rheology 57 (1) (2013) 27-70.

[49] S. Hormozi, G. Dunbrack, I. Frigaard, Visco-plastic sculpting, Physics of Fluids 26 (9) (2014) 093101.

[50] B. Gueslin, L. Talini, Y. Peysson, Sphere settling in an aging yield stress fluid: link between the induced flows and the rheological behavior, Rheologica acta 48 (9) (2009) 961.

[51] Y. Holenberg, O. M. Lavrenteva, U. Shavit, A. Nir, Particle tracking velocimetry and particle image velocimetry study of the slow motion of rough and smooth solid spheres in a yield-stress fluid, Physical Review E 86 (6) (2012) 066301.

[52] F. Ahonguio, L. Jossic, A. Magnin, Influence of surface properties on the flow of a yield stress fluid around spheres, Journal of Non-Newtonian Fluid Mechanics 206 (2014) 57-70. 
[53] A. Van Dinther, C. Schroën, F. Vergeldt, R. Van der Sman, R. Boom, Suspension flow in microfluidic devicesa review of experimental techniques focussing on concentration and velocity gradients, Advances in colloid and interface science 173 (2012) 23-34.

[54] G. Ovarlez, F. Mahaut, S. Deboeuf, N. Lenoir, S. Hormozi, X. Chateau, Flows of suspensions of particles in yield stress fluids, Journal of rheology 59 (6) (2015) 1449-1486.

[55] T. J. Heindel, A review of x-ray flow visualization with applications to multiphase flows, Journal of Fluids Engineering 133 (7) (2011) 074001.

[56] M. Gholami, N. Lenoir, D. Hautemayou, G. Ovarlez, S. Hormozi, Timeresolved $2 \mathrm{~d}$ concentration maps in flowing suspensions using x-ray, Journal of rheology under review.

[57] R. L. Powell, Experimental techniques for multiphase flows, Physics of fluids 20 (4) (2008) 040605.

[58] S. Bhavaraju, R. Mashelkar, H. Blanch, Bubble motion and mass transfer in non-newtonian fluids: Part i. single bubble in power law and bingham fluids, AIChE Journal 24 (6) (1978) 1063-1070.

[59] A. Potapov, R. Spivak, O. M. Lavrenteva, A. Nir, Motion and deformation of drops in bingham fluid, Industrial \& engineering chemistry research 45 (21) (2006) 6985-6995.

[60] J. Tsamopoulos, Y. Dimakopoulos, N. Chatzidai, G. Karapetsas, M. Pavlidis, Steady bubble rise and deformation in newtonian and viscoplastic fluids and conditions for bubble entrapment, Journal of Fluid Mechanics 601 (2008) 123-164.

[61] J. P. Singh, M. M. Denn, Interacting two-dimensional bubbles and droplets in a yield-stress fluid, Physics of Fluids 20 (4) (2008) 040901.

[62] Y. Dimakopoulos, M. Pavlidis, J. Tsamopoulos, Steady bubble rise in herschel-bulkley fluids and comparison of predictions via the augmented lagrangian method with those via the papanastasiou model, Journal of Non-Newtonian Fluid Mechanics 200 (2013) 34-51.

[63] O. M. Lavrenteva, Y. Holenberg, A. Nir, Motion of viscous drops in tubes filled with yield stress fluid, Chemical Engineering Science 64 (22) (2009) $4772-4786$.

[64] Y. Holenberg, O. M. Lavrenteva, A. Nir, Interaction of viscous drops in a yield stress material, Rheologica acta 50 (4) (2011) 375-387.

[65] A. Maleki, S. Hormozi, A. Roustaei, I. Frigaard, Macro-size drop encapsulation, Journal of Fluid Mechanics 769 (2015) 482-521. 
[66] E. Chaparian, I. A. Frigaard, Yield limit analysis of particle motion in a yield-stress fluid, Journal of Fluid Mechanics 819 (2017) 311-351.

[67] L. Jossic, A. Magnin, Drag and stability of objects in a yield stress fluid, AIChE journal 47 (12) (2001) 2666-2672.

[68] X. Chateau, G. Ovarlez, K. L. Trung, Homogenization approach to the behavior of suspensions of noncolloidal particles in yield stress fluids, Journal of Rheology 52 (2) (2008) 489-506.

[69] F. Mahaut, X. Chateau, P. Coussot, G. Ovarlez, Yield stress and elastic modulus of suspensions of noncolloidal particles in yield stress fluids, Journal of Rheology 52 (1) (2008) 287-313.

[70] G. Ovarlez, F. Bertrand, P. Coussot, X. Chateau, Shear-induced sedimentation in yield stress fluids, Journal of Non-Newtonian Fluid Mechanics 177 (2012) 19-28.

[71] G. Ovarlez, N. Roussel, A physical model for the prediction of lateral stress exerted by self-compacting concrete on formwork, Materials and Structures 39 (2) (2006) 269-279.

[72] T.-S. Vu, G. Ovarlez, X. Chateau, Macroscopic behavior of bidisperse suspensions of noncolloidal particles in yield stress fluids, Journal of Rheology 54 (4) (2010) 815-833.

[73] S. Dagois-Bohy, S. Hormozi, É. Guazzelli, O. Pouliquen, Rheology of dense suspensions of non-colloidal spheres in yield-stress fluids, Journal of Fluid Mechanics 776 .

[74] B. Metzger, J. E. Butler, Clouds of particles in a periodic shear flow, Physics of Fluids 24 (2) (2012) 021703.

[75] P. Pham, Origin of shear-induced diffusion in particulate suspensions: Crucial role of solid contacts between particles, Ph.D. thesis, University of Florida (2016).

[76] M. Souzy, Mélange dans les suspensions de particules cisaillées à bas nombre de reynolds, Ph.D. thesis, Aix Marseille Université (2016).

[77] M. Souzy, H. Lhuissier, E. Villermaux, B. Metzger, Stretching and mixing in sheared particulate suspensions, Journal of Fluid Mechanics 812 (2017) 611-635.

[78] N. J. Balmforth, I. A. Frigaard, G. Ovarlez, Yielding to stress: recent developments in viscoplastic fluid mechanics, Annual Review of Fluid Mechanics 46 (2014) 121-146.

[79] G. Ovarlez, S. Cohen-Addad, K. Krishan, J. Goyon, P. Coussot, On the existence of a simple yield stress fluid behavior, Journal of Non-Newtonian Fluid Mechanics 193 (2013) 68-79. 
[80] I. A. Gutowski, D. Lee, J. R. de Bruyn, B. J. Frisken, Scaling and mesostructure of carbopol dispersions, Rheologica acta 51 (5) (2012) 441450.

[81] D. Lee, I. A. Gutowski, A. E. Bailey, L. Rubatat, J. R. de Bruyn, B. J. Frisken, Investigating the microstructure of a yield-stress fluid by light scattering, Physical Review E 83 (3) (2011) 031401.

[82] J. Piau, Carbopol gels: Elastoviscoplastic and slippery glasses made of individual swollen sponges: Meso-and macroscopic properties, constitutive equations and scaling laws, Journal of non-newtonian fluid mechanics 144 (1) (2007) 1-29.

[83] S. Inc, Jaguar, product guide for personal care solutions (2015).

[84] P. Uhlherr, J. Guo, C. Tiu, X.-M. Zhang, J.-Q. Zhou, T.-N. Fang, The shear-induced solid-liquid transition in yield stress materials with chemically different structures, Journal of Non-Newtonian Fluid Mechanics 125 (2-3) (2005) 101-119.

[85] P. Coussot, Rheometry of pastes, suspensions, and granular materials: applications in industry and environment, John Wiley \& Sons, 2005.

[86] D. Risica, A. Barbetta, L. Vischetti, C. Cametti, M. Dentini, Rheological properties of guar and its methyl, hydroxypropyl and hydroxypropylmethyl derivatives in semidilute and concentrated aqueous solutions, Polymer 51 (9) (2010) 1972-1982.

[87] D. Szopinski, W.-M. Kulicke, G. A. Luinstra, Structure-property relationships of carboxymethyl hydroxypropyl guar gum in water and a hyperentanglement parameter, Carbohydrate polymers 119 (2015) 159-166.

[88] T. Peng, Detect circles with various radii in grayscale image via hough transform

11 URL https://www.mathworks.com/matlabcentral/fileexchange/ 9168-detect-circles-with-various-radii-in-grayscale-image-via-hough-transform

[89] R. O. Duda, P. E. Hart, Use of the hough transformation to detect lines and curves in pictures, Communications of the ACM 15 (1) (1972) 11-15.

[90] J. K. Sveen, An introduction to matpiv v. 1.6. 1, Preprint series. Mechanics and Applied Mathematics http://urn. nb. no/URN: NBN: no-23418.

[91] L. G. Leal, Advanced transport phenomena: fluid mechanics and convective transport processes, Cambridge University Press, 2007.

[92] C. Darabaner, S. Mason, Particle motions in sheared suspensions xxii: Interactions of rigid spheres (experimental), Rheologica Acta 6 (3) (1967) $273-284$. 
[93] H. Fahs, G. Ovarlez, X. Chateau, Pair-particle trajectories in a shear flow of a bingham fluid, Journal of Fluid Mechanics under review.

[94] F. Snijkers, R. Pasquino, J. Vermant, Hydrodynamic interactions between two equally sized spheres in viscoelastic fluids in shear flow, Langmuir 29 (19) (2013) 5701-5713. 